\title{
Nano-array based Monolithic Catalysts: Concept, Rational Materials Design and Tunable Catalytic Performance
}

Zheng Ren, Yanbing Guo and Pu-Xian Gao*

Department of Materials Science and Engineering \& Institute of Materials Science

University of Connecticut, Storrs, CT 06269-3136, USA

*Email: puxian.gao@ims.uconn.edu

\begin{abstract}
Monolithic catalysts, also known as structured catalysts, represent an important catalyst configuration widely used in automotive, chemical, and energy industries. However, several issues associated with washcoat based monolithic catalyst preparation are ever present, such as compromised materials utilization efficiency due to a less-than-ideal wash coating process, difficulty in precise and optimum microstructure control and lack of structure-property correlation. In this mini-review, we introduce the concept of nano-array catalyst, a new type of monolithic catalyst featuring high catalyst utilization efficiency, good thermal/mechanical robustness, and catalytic performance tunability. A comprehensive overview is presented with detailed discussion of the strategies for nano-array catalyst preparation and rational catalytic activity adjustment enabled by the well-defined nano-array geometry. Specifically their scalable fabrication processes are reviewed in conjunction with discussion of their various catalytic oxidation reaction performances at low temperature. We hope this review will serve as a timely and useful research guide for rational design and utilization of the new type of monolithic catalysts.
\end{abstract}

Keywords: monolithic catalyst, nano-arrays, rational materials design, scalable synthesis, structure-property relations, low temperature oxidation

\section{Introduction}

Monolith structure represents an important configuration of reactors in many applications such as fine chemical processing, environmental catalysis and clean energy combustion. ${ }^{1-9}$ The monolith 
structure can be made by various types of materials such as metals, ceramics, and polymers. Amongst them, ceramic monolith made of cordierite $\left(2 \mathrm{MgO} \cdot 2 \mathrm{Al}_{2} \mathrm{O}_{3} \cdot 5 \mathrm{SiO}_{2}\right)$ is one of the major types of monoliths used as catalytic reactors for automotive emission control, ${ }^{3,10-12}$ diesel particulate filter (DPF), ${ }^{13-15}$ indoor air purification ${ }^{16}$ and water filtration ${ }^{17-19}$ due to its low thermal expansion coefficient, high thermal shock resistance, relatively high porosity, low weight but good mechanical durability. ${ }^{3,20}$ Figure 1 shows a photograph of cordierite ceramic monolith purchased from Corning Incorporated. The cordierite monolith consists of parallel channels that extend throughout the substrate. The geometric characteristics of this ceramic monolith are described by several parameters including cell density, hydraulic diameter, wall thickness and open frontal area. If the cell spacing $\mathrm{L}$ is defined as the distance between the centers of adjacent channels, the hydraulic diameter is thus $D=L-t$ where $t$ represents the wall thickness. The cell density $\mathrm{N}=1 / \mathrm{L}^{2}$ is defined as the number of cells per unit cross-sectional area. The open frontal area $O F A=\frac{(L-t)^{2}}{L^{2}}=D^{2} N$ is the fraction of the open cross-sectional region in an individual cell. The monolith configuration and the ceramic cordierite enable several advantages in gas phase reactions especially in automotive emission control. The high thermal shock resistance and sufficient mechanical strength allow it to survive under various automotive operation conditions. Furthermore, the low pressure drop to surface area ratio enabled by high cell density and large OFA is beneficial to handle large flow rate with less energy consumption which successfully reduce the cost of exhaust after-treatment. The large OFA also favors the treatment of stream with particulate and dust for DPF application to remove soot particles in diesel engines.

Despite all the benefits the monolith configuration is able to provide, challenges remain for preparation of monolithic catalysts. Typically the catalyst preparation requires a uniform coating upon the monolithic substrate of either ready-made catalysts (e.g. zeolites) which do not need specific catalytic supports or both support and catalytic active materials. ${ }^{21}$ For example, washcoating of $\gamma-\mathrm{Al}_{2} \mathrm{O}_{3}, \mathrm{SiO}_{2}$ or carbon as the catalyst support is usually the first step of the monolithic catalyst fabrication followed by the dispersion of active noble metal ( $\mathrm{Pt}, \mathrm{Rh}$ or $\mathrm{Pd})$ nanoparticles. Two typical strategies, pore-filling and slip-casting, are widely used for this wash coating procedure. The pore filling refers to the filling of pores in the monolith ceramics with support materials by colloidal coating which features strong interaction between the support and 
the substrate. Although mono-dispersed $\mathrm{Al}_{2} \mathrm{O}_{3}$ or $\mathrm{SiO}_{2}$ colloidal suspension is easily to be prepared by wet chemical synthesis and the pore-filling process ensures the good adherence since the support materials is confined in the pores, the loading amount is very limited given the relatively small pore volume and low surface area of the cordierite monolith. ${ }^{3}$ The slip-casting strategy, also known as slurry coating, is usually employed to increase the materials loading. Since the layer is thicker and most of the materials are not confined to the pores, the adherence to the monolith must be enhanced by adding binders. Such slurry coating sacrifices the catalyst utilization efficiency as a consequence of large amount of catalyst loading. It also adds complexity to the materials processing by the addition of binders because the viscosity of the slurry needs to be carefully controlled and any wrongly prepared slurry precursor will lead to poor adherence to the substrate. In addition, a number of repeated coating procedures are often required to ensure a uniform coverage. Therefore the traditional monolithic catalyst configuration faces several challenges. Firstly, the significant amount of materials loading imposes great pressure on the noble metal supply which makes the catalytic converters rather expensive for automotive emission control. Secondly, the less-than-ideal wash-coating process usually does not ensure uniform catalyst deposition with precisely and optimally controlled microstructures, therefore compromises the materials utilization efficiency. Lastly, the random structural arrangement of the wash-coated materials renders difficulty to well correlate catalyst structure, porosity, orientation and the relevant gas-solid interactions with catalyst performance and it is thus no easy task for the rational catalyst design.

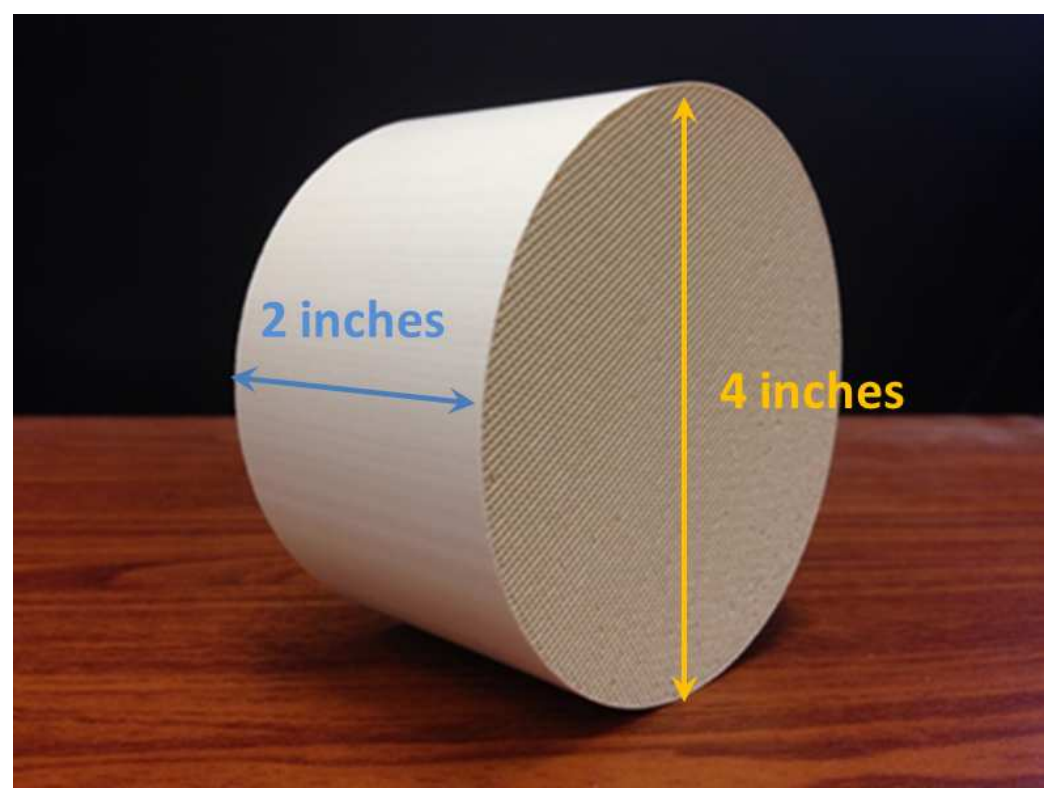


Figure 1. A photograph of a cordierite monolith purchased from Corning Incorporated.

To meet the above challenges of traditional wash-coat based monolithic catalysts, in the past few years, a distinct monolithic catalyst configuration, nanostructure array (nano-array) based monolithic catalysts, has been invented and demonstrated in our research group by in-situ growing hierarchically arranged arrays of various types of nanostructures such as nanowires, nanorods and nanotubes onto bare channeled monolithic substrates. ${ }^{22-24}$ The unique in-situ solution based self-assembly and integration process and ordered nano-arrays instead of washcoated catalyst layers on the three-dimensional (3D) monolith substrate differentiate the nanoarray catalyst from the traditional monolithic catalyst in terms of fabrication and structural characteristics. Furthermore, the well-defined structural and chemical characteristics conveyed by this new type of structured catalysts have demonstrated potential figures of merits in excellent robustness, high materials utilization efficiency, as well as tunable catalytic functions toward various gas phase reactions at low temperature. The gas phase reactions include $\mathrm{CO}$ oxidation, hydrocarbon oxidation, as well as nitric oxide oxidation and reduction. In this article, this new concept of nano-array based monolithic catalysts will be introduced with their latest research progress comprehensively reviewed. Specifically, our discussion will be focused on reviewing the progress on the rational catalyst materials design and preparation, and highlighting the demonstrated figures of merit in high robustness, materials utilization efficiency and tunable catalytic reaction activity toward various catalytic oxidations at low temperature.

\section{Concept of Nano-array based Monolithic Catalysts}

The typical structural configuration of nano-array based monolithic catalysts is illustrated in Figure 2, where nano-arrays such as nanowire arrays are distributed uniformly on the channel surfaces of cordierite monolith. ${ }^{22}$ The nano-arrays can either act as the support for catalytic active materials or function as the catalysts themselves. The height of nanowire arrays is usually less than $10 \mu \mathrm{m}$, much thinner than the wash-coat thickness $(\sim 40-100 \mu \mathrm{m})$ in traditional washcoated structured catalysts, thus greatly improve the catalyst utilization efficiency by enabled shorter diffusion distance and low materials usage. The mass loading of these nano-arrays only constitutes $5 \% \sim 15 \%$ of the monolithic catalyst. Using CO oxidation as the probe reaction we have discovered the nano-array configuration reduces the materials utilization by an order of magnitude without sacrificing the catalytic activity. For example, $1 \% \mathrm{Pt}$ supported on $\mathrm{ZnO}$ nano- 
arrays ( $\sim 0.1 \mathrm{mg} \mathrm{Pt}$ and $\sim 10.0 \mathrm{mg} \mathrm{ZnO})$ is able to achieve complete $\mathrm{CO}$ oxidation below $300{ }^{\circ} \mathrm{C}$ at a relatively large space velocity of $\sim 50,000 \mathrm{~h}^{-1}$.

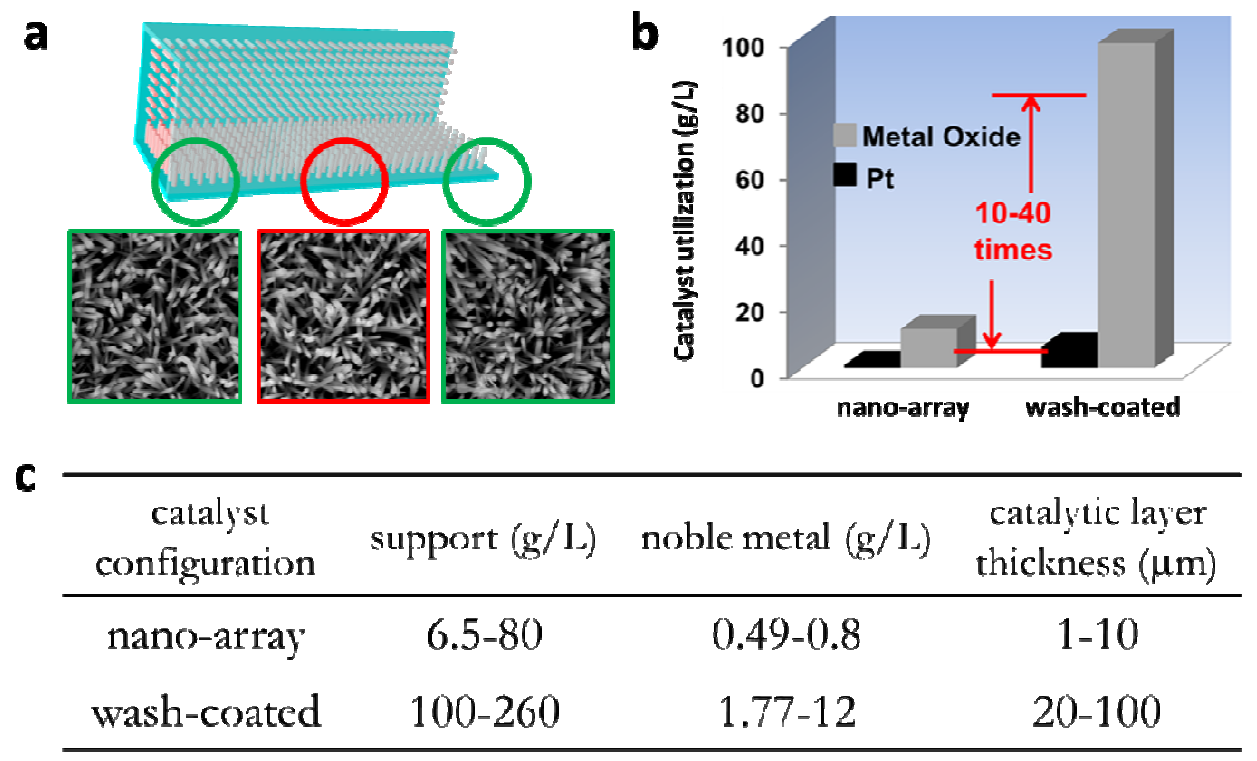

Figure 2. a) Schematic and micrographic illustration of the nanostructure arrays in-situ grown on the individual channel surface of the monolithic substrate; b) histogram comparison and c) summary table of catalyst utilization efficiencies for nano-array based monolithic catalysts and washcoated ones. Reprinted with permission from ref. 22.

Other advantageous features of nano-array catalyst over wash-coated particulate form catalyst include the high surface area, thermal stability and mechanical robustness. As shown in Figure 3, the morphology of the nano-arrays after prolonged high temperature aging does not vary much, with small mass loss observed in the first 24 hours. The crystal structures of aged metal oxides nano-arrays retained without phase segregation as revealed by XRD analysis. The measured surface area loss of typical nano-arrays after 100 hours $800{ }^{\circ} \mathrm{C}$ annealing was much smaller than their counterparts of wash-coated catalysts. For instance, the surface area loss of $\mathrm{ZnO}$ nanoarrays was found to be only $5 \%$ while that of $\mathrm{ZnO}$ wash-coated powders was over $50 \%$. $\mathrm{TiO}_{2}$ nano-arrays suffered $40 \%$ surface area loss but the $\mathrm{TiO}_{2}$ powders lost over $80 \%$ of the surface area. The nano-arrays also demonstrate good mechanical integrity under fast air flux $(50 \mathrm{~L} / \mathrm{min})$ for 10 days, featuring little morphology destruction and negligible weight loss. All these intriguing properties as well as the high catalytic activity highlighted in sections 4 and 5 make 
nano-array based monolithic catalysts a cost-effective and promising class of structured catalyst candidates for various catalytic reactions in automotive emission control, clean energy combustion, and pollutant abatement.

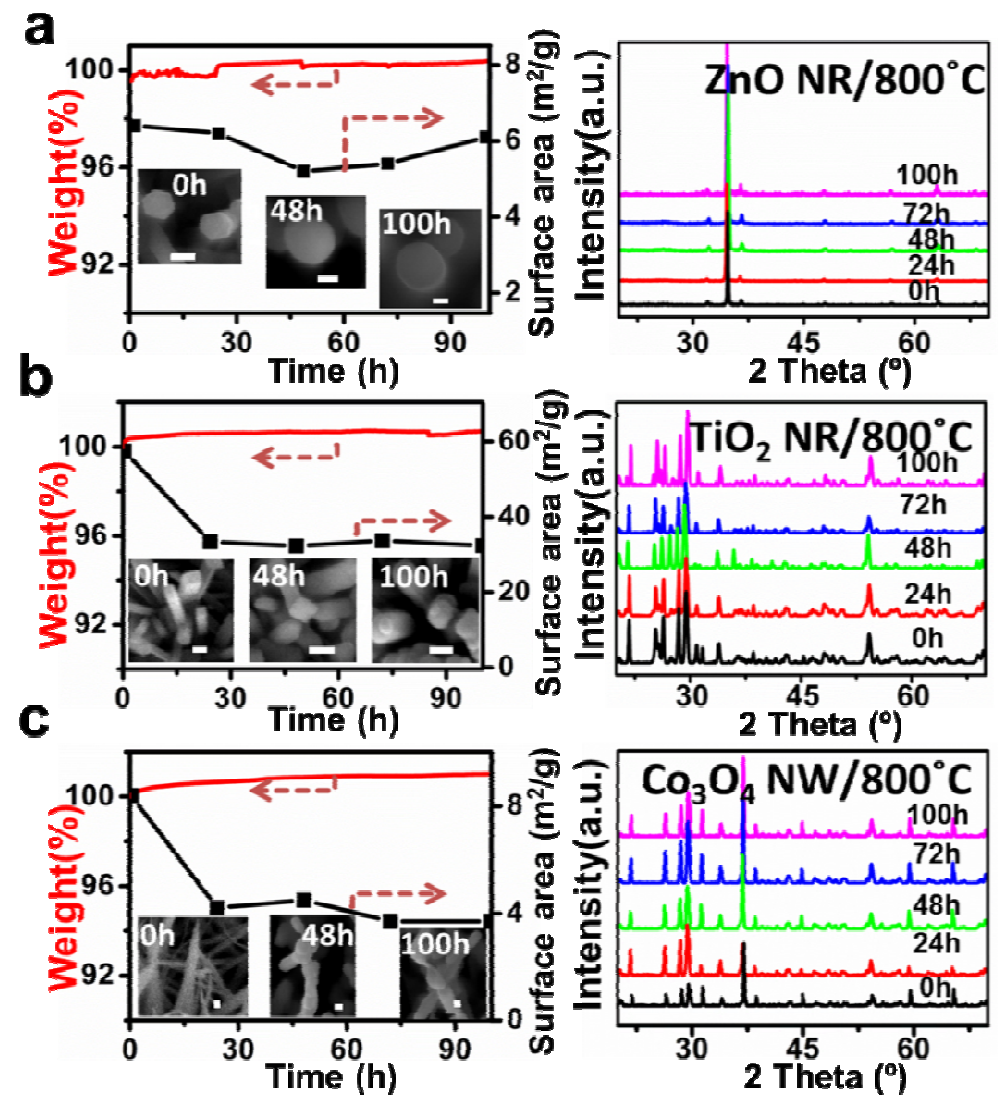

Figure 3. Morphology, weight loss, surface area loss and crystal structure evolution of typical nano-array based monolithic catalysts after 100 hours' ambient annealing at $800{ }^{\circ} \mathrm{C}$. Reprinted with permission from ref. 22.

\section{Materials Design and Processing}

In this section we will discuss the synthetic strategies for preparing the nano-array based monolithic catalyst. Starting with the nano-arrays assembled on the planar substrate we will introduce the wet chemical growth of transition metal oxide and complex oxide nanostructures. This serves the foundation for the hierarchical assembly of nano-arrays in the 3D fashion. In the very last part of this section, the industrial scalability of nano-array based monolithic catalysts will be discussed, which is of significant implication for nano-array catalysts technology commercialization and utilization in the near future. 


\subsection{Two dimensional assembly}

Hydrothermal synthesis is a facile and widely used approach to grow nanowire arrays on planar substrates. The one-pot hydrothermal synthesis is a binder-free and environmentally benign process to grow nanostructures with controlled geometry and orientation. The grown nano-arrays usually have good adherence to the deposition substrate since the crystal growth starts with the nucleation on the substrate. For example, Figures $4 \mathrm{a}$ and $4 \mathrm{~b}$ display the hydrothermally grown $\mathrm{ZnO}$ and $\mathrm{TiO}_{2}$ nano-arrays on the planar silicon substrate respectively. ${ }^{25,26}$ The single crystalline nanowires are uniformly aligned normal to the substrate surface. The length of nanowires is less than $1 \mu \mathrm{m}$ and their diameter is smaller than $150 \mathrm{~nm}$. The growth of $\mathrm{ZnO}$ nano-arrays follows a hydrothermal process mediated by hexamethylenetetramine (HMT), a bidentate Lewis base that bridges two $\mathrm{Zn}^{2+}$ ions. ${ }^{27}$ The selective HMT molecule attaching to the nonpolar surface of $\mathrm{ZnO}$ facilitates the anisotropic growth along [0001] direction to form one-dimensional nanowires. $\mathrm{TiO}_{2}$ nano-arrays are fabricated by the hydrolysis of titanium precursors in the acidic aqueous solution where sodium chloride acts as the buffer. The nanowire growth starts with the large scale nucleation on the substrate and clusters of nanowires quickly develop at reaction

temperature of $180{ }^{\circ} \mathrm{C} .{ }^{28}$ As reaction proceeds these clusters get densely packed leading to large scale nano-arrays. The crystal phase of $\mathrm{TiO}_{2}$ nano-arrays has been discovered to be brookite. The phase and morphology control of $\mathrm{TiO}_{2}$ nano-arrays still requires further investigation. 

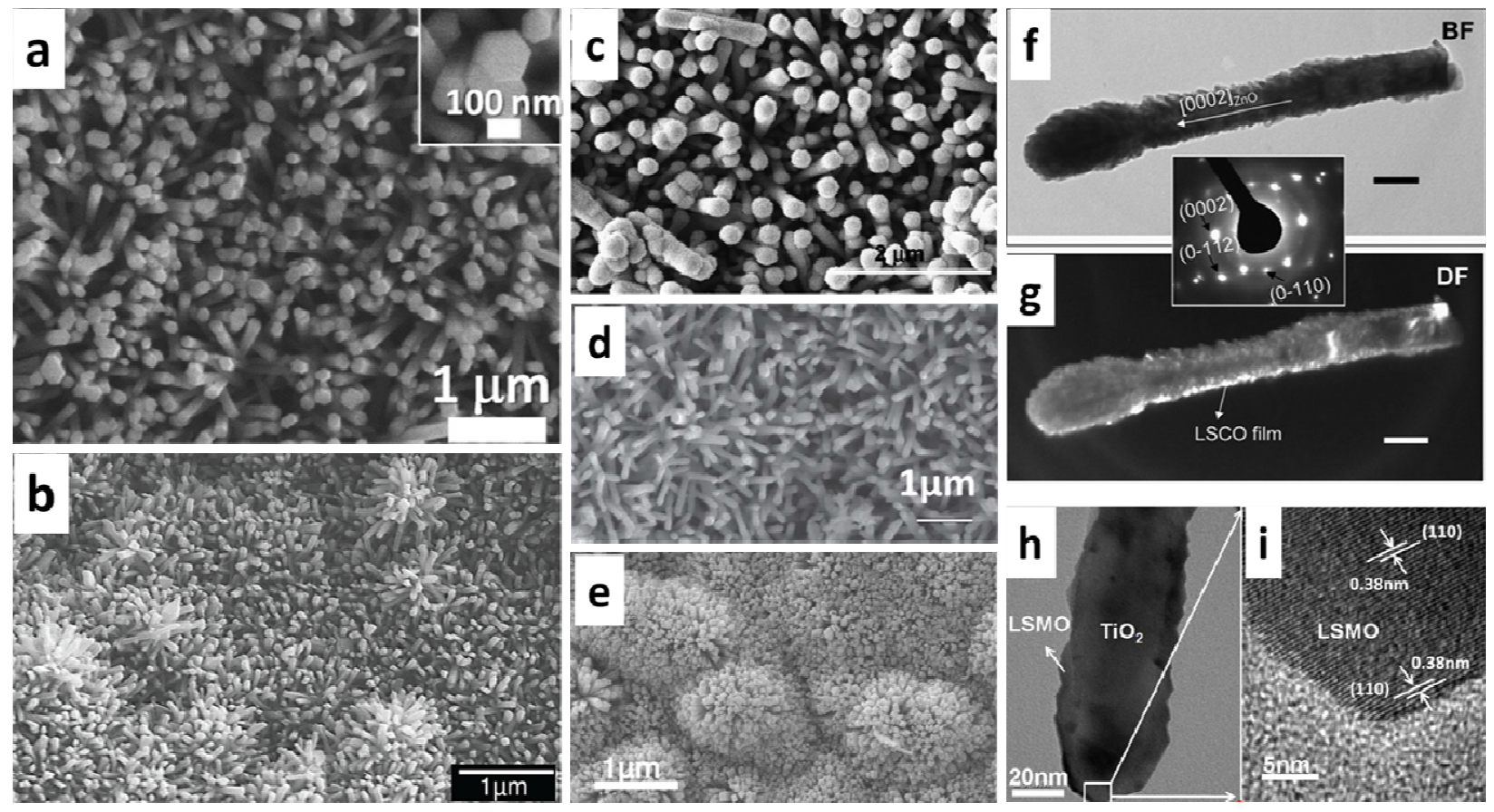

Figure 4. Electron microscopy characterization of various metal oxide nanorod arrays grown on planar silicon substrates. SEM images of a) $\mathrm{ZnO}$ nano-arrays; b) $\mathrm{TiO}_{2}$ nano-arrays; c) $\mathrm{ZnO} /(\mathrm{La}, \mathrm{Sr}) \mathrm{CoO}_{3}$ nano-arrays; d) $\mathrm{ZnO} / \mathrm{CeO}_{2}$ nano-arrays. f) bright-field and g) dark field TEM images of $\mathrm{ZnO} /(\mathrm{La}, \mathrm{Sr}) \mathrm{CoO}_{3}$ nanorods; h) and i) $\mathrm{TEM}$ images of $\mathrm{TiO}_{2} /(\mathrm{La}, \mathrm{Sr}) \mathrm{MnO}_{3}$ core-shell nanorods. Reprinted with permission from refs. 25, 26, and 29.

By using magnetron sputtering method, a thin layer of catalytically active materials such as flurites (e.g., $\mathrm{CeO}_{2}$ ) and perovskites (e.g., $\left.(\mathrm{La}, \mathrm{Sr}) \mathrm{CoO}_{3}\right)$ can be coated onto the nano-array surfaces to achieve core-shell composite nanomaterials system. ${ }^{29}$ These composite nano-arrays combine the high surface area, an important merit by nano-arrays, with the high activity of catalytic shell layer making them promising candidates for various catalytic reactions. This coreshell composite nano-array design strategy can potentially enhance the catalyst efficiency by providing high surface area and low materials usage.

\subsection{Three dimensional monolithic integration}

We further applied the hydrothermal synthesis to the in-situ growth of metal oxide nano-arrays on commercial 3D cordierite honeycomb substrates. In addition to the above-mentioned $\mathrm{ZnO}$ and $\mathrm{TiO}_{2}$ nano-arrays, $\mathrm{Co}_{3} \mathrm{O}_{4}$ nanowires arrays and $\mathrm{CeO}_{2}$ nanotube arrays have been successfully prepared. ${ }^{22}$ Figure 5a illustrates the fabrication process for various nano-arrays on 3D cordierite 
and stainless steel honeycomb substrates. The substrate was $\sim 1$ inch in diameter and 0.5 inch in channel length. The as-prepared monolithic nano-array catalyst demonstrates the feasibility of direct nano-array integration upon the 3D substrate by solution chemistry on the laboratory scale. By controlling the reaction chemistry, the length of $\mathrm{ZnO}$ nanowires can be adjusted in the range of 1-5 $\mu \mathrm{m}$ while $\mathrm{TiO}_{2}$ nanorods have the length tuned from $500 \mathrm{~nm}$ to $1 \mu \mathrm{m}$. Given the direct hydrothermal synthesis of $\mathrm{CeO}_{2}$ nano-arrays is challenging, a templated method has been developed to prepare $\mathrm{CeO}_{2}$ nanotubes. The hydrothermally grown $\mathrm{ZnO}$ nanowires were used as the template and $\mathrm{CeO}_{2}$ layers were subsequently deposited to the $\mathrm{ZnO}$ surface to form core-shell nanostructures. Since $\mathrm{ZnO}$ is easily to react with hydrogen and zinc metal has a relatively low sublimation point, the $\mathrm{ZnO}$ core can be removed by hydrogen treatment at high temperature, resulting in the formation of $\mathrm{CeO}_{2}$ nanotubes. The porous $\mathrm{Co}_{3} \mathrm{O}_{4}$ nanowire arrays prepared by urea hydrolysis during hydrothermal process have a diameter of $\sim 100 \mathrm{~nm}$ and a length of $\sim 10 \mu \mathrm{m}$. The evolution of nanowire morphology follows the crystal-splitting mechanism as detailed in our earlier report ${ }^{34}$. The porous nature of $\mathrm{Co}_{3} \mathrm{O}_{4}$ nanowires gives rise to the high specific surface area which is beneficial for catalytic reactions. The electron microscopy characterization in Figure 5 shows the uniform coverage of nano-arrays with controlled morphology and orientation. 
a
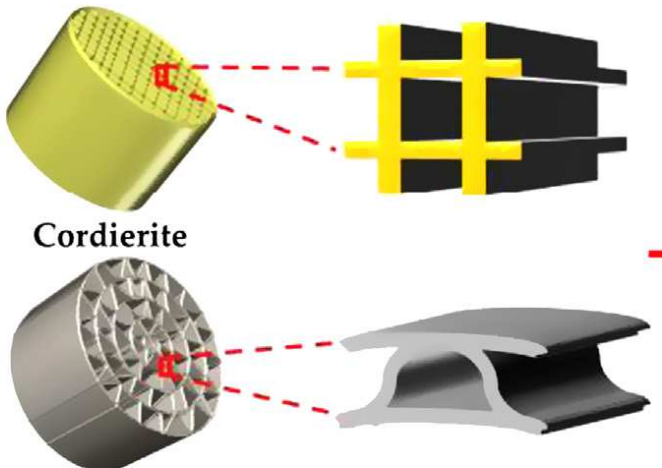

Stainless steel
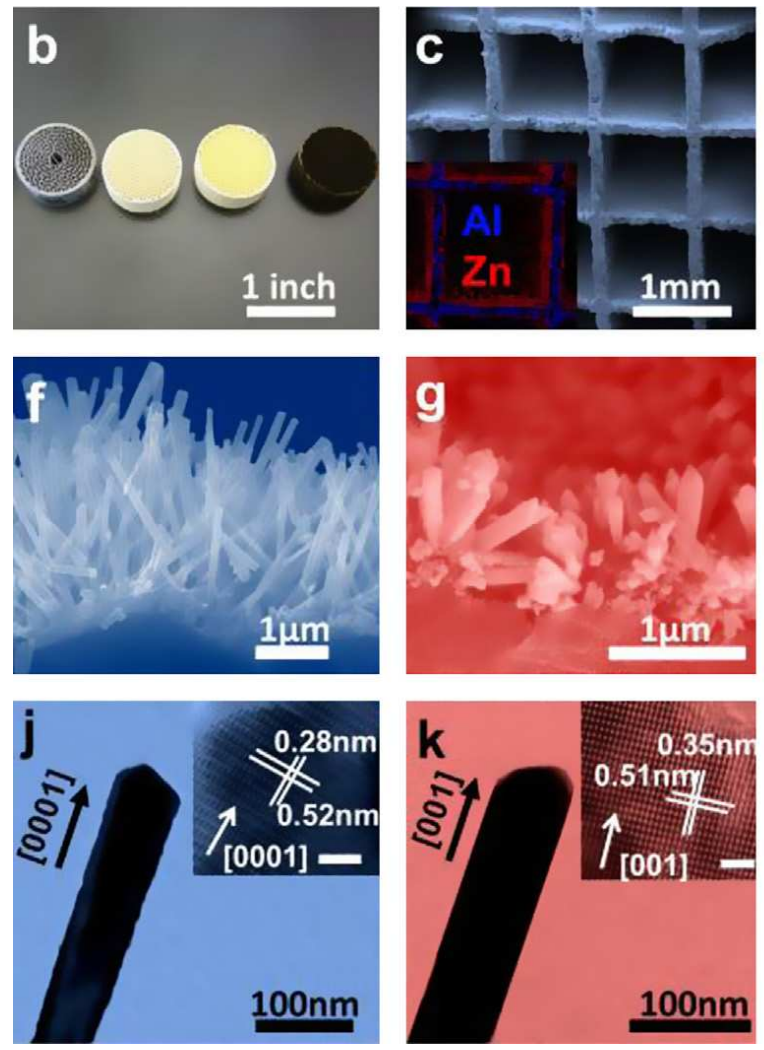

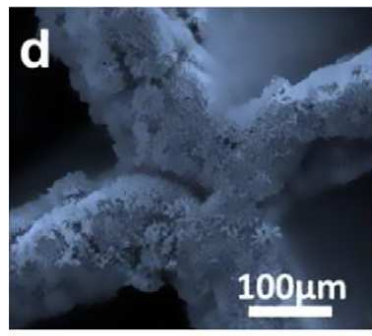

In-situ 3D

rational assembly

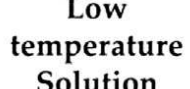

Solution

method
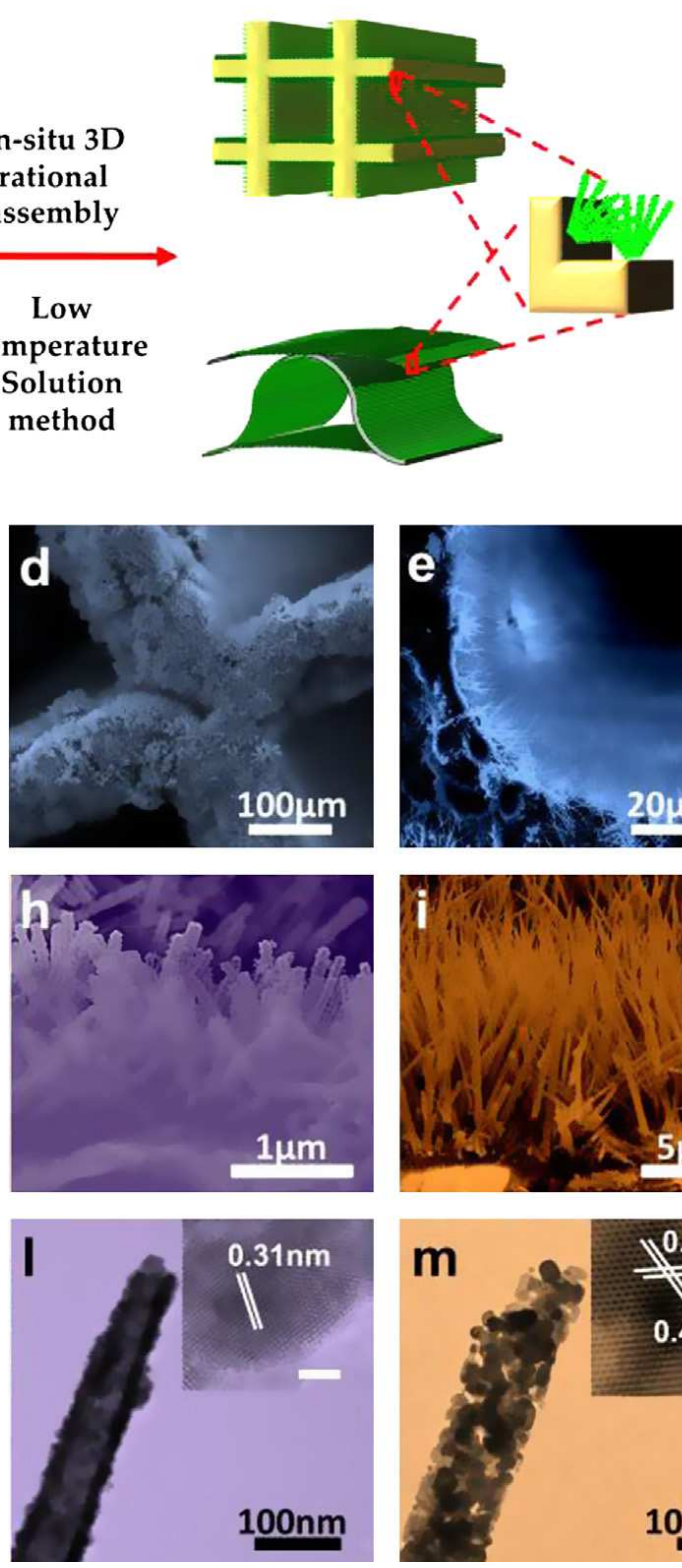
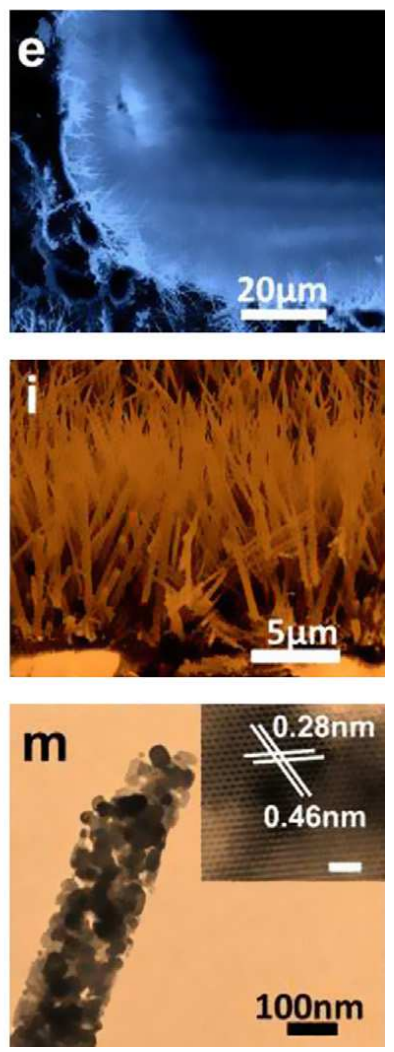

Figure 5. a) Schematic illustration for the $3 \mathrm{D}$ integration of nano-arrays onto the monolithic cordierite and stainless steel substrate by a hydrothermal approach; b) Photographs of monolithic nano-array catalysts with various nano-arrays deposited; c) low magnification SEM image of $\mathrm{ZnO}$ nano-array catalyst with inset showing the element distribution; d-e) low magnification top and cross-sectional SEM images of nano-arrays; f-i) cross-sectional SEM images of f) ZnO nanowire arrays; g) $\mathrm{TiO}_{2}$ nanorod arrays; h) $\mathrm{CeO}_{2}$ nanotube arrays; i) $\mathrm{Co}_{3} \mathrm{O}_{4}$ nanowire arrays; $\mathrm{j}$ $\mathrm{m}$ ) corresponding TEM characterization of nano-arrays shown in f-i). Reprinted with permission from ref. 22. 


\subsection{Scalable fabrication}

Previously, the feasibility of hydrothermal synthesis for 3D integration of nano-arrays into the channeled monolithic substrates has been proven by demonstrating monolithic nano-array catalyst of small size. However, the industrially relevant manufacturing of nano-array based monolithic catalysts is an indispensable step toward practical application in catalyst industry. A major hurdle for the large scale fabrication is the difficulty of solution precursor mass transfer and uniform access throughout the space confined channel surfaces during the hydrothermal nanostructure integration into monolithic substrates with long channels. It becomes especially challenging when the reaction temperature is low. For example, the growth of $\mathrm{ZnO}$ and $\mathrm{Co}_{3} \mathrm{O}_{4}$ nanowires only requires $90^{\circ} \mathrm{C}$ or even lower in the aqueous media. The low reaction temperature makes the precursor solution relatively stagnant during the synthesis and may not favor the uniform mass transport throughout the long monolithic channels.

To facilitate the mass transport during the low temperature hydrothermal synthesis, we have successfully introduced the mechanical agitation enabled by simple magnetic stirring and have achieved uniform coverage of nano-arrays structures throughout the extended monolithic channels. ${ }^{30}$ Figure 6 shows the $\mathrm{ZnO}$ nano-array growth with and without magnetic stirring. It is clearly observed that the nano-arrays coverage in the center region of cordierite channels is greatly improved when the magnetic stirring is introduced in the synthesis. Only sparsely distributed $\mathrm{ZnO}$ nano-arrays exist in the same region without stirring, which implies the mechanical agitation helps promote the mass transfer during the hydrothermal growth. Computational fluidic dynamics (CFD) simulation provides more insights of the magnetic stirring effect. Specifically the monolith was placed at one side of the reactor while the rotating magnetic bar was on the other side so that the stirring would generate constant flow through the cordierite channels. The CFD results reveal the existence of an oscillating pressure difference between two ends of each individual channel. Such oscillating pressure drop drives the precursor solution to flow back and forth within cordierite channels and thus enhance the mass transfer. 

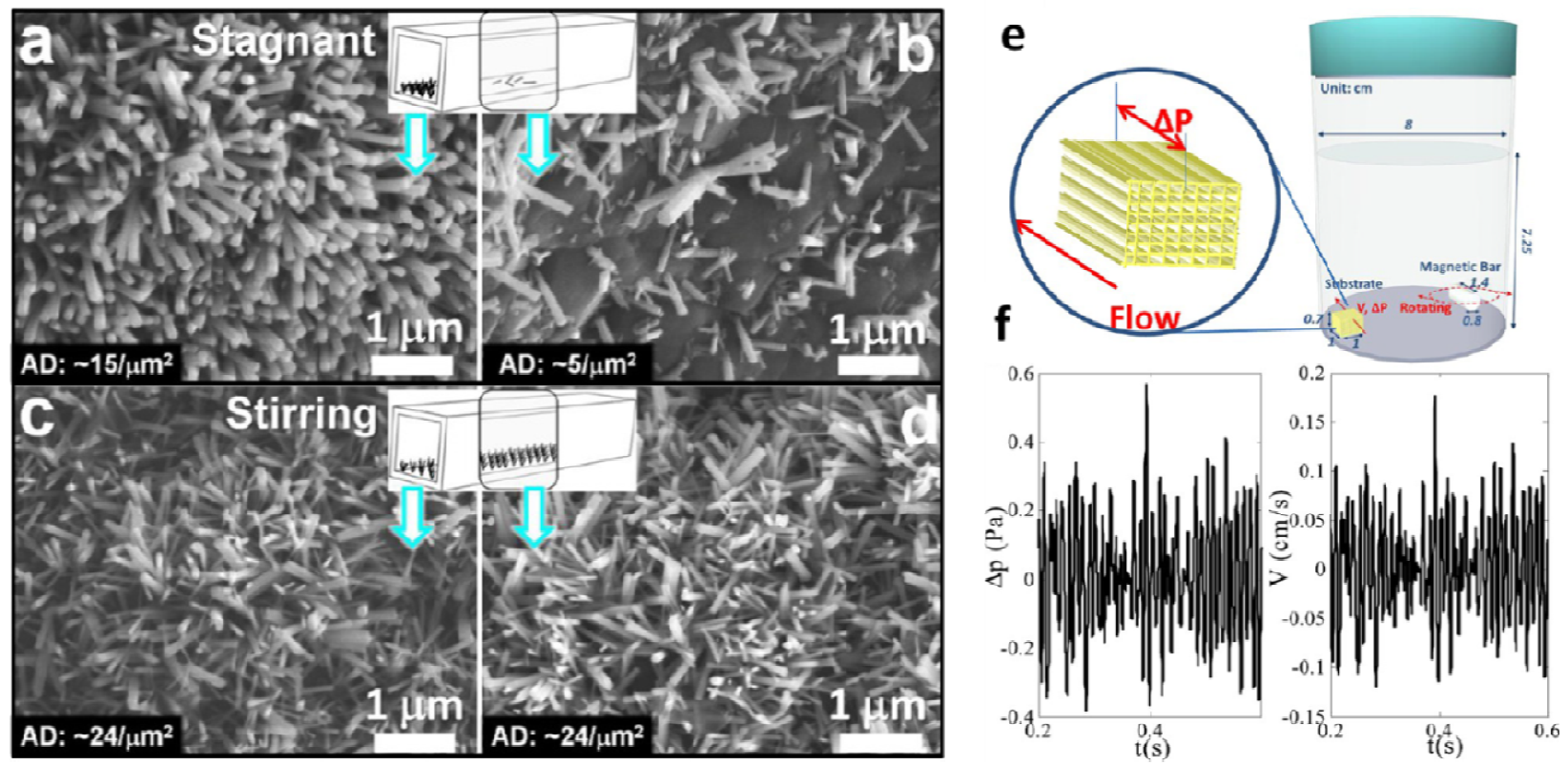

Figure 6. Mechanical agitation assisted hydrothermal growth of $\mathrm{ZnO}$ nano-arrays. a-d) SEM images of the as-grown nano-arrays at the end and center regions of cordierite channels after synthesis with and without mechanical agitation; e-f) computational fluidic dynamics (CFD) modeling for the introduced mechanical agitation by magnetic stirring. Reprinted with permission from ref. 30 .

Large scale $\mathrm{Co}_{3} \mathrm{O}_{4}$ nano-array monolithic catalyst has been successfully fabricated with $\mathrm{Co}_{3} \mathrm{O}_{4}$ nanowire arrays uniformly deposited on cordierite monoliths of 4 inches in diameter and 2 inches in channel length, an industrially relevant size that can be directly used. ${ }^{31}$ As shown in Figure 8, the mechanical agitation introduced by magnetic stirring has resulted in good coverage of $\mathrm{Co}_{3} \mathrm{O}_{4}$ nanowire arrays all around the monolith substrates. SEM characterization of nano-arrays in different regions in the substrate confirms the $\mathrm{Co}_{3} \mathrm{O}_{4}$ nanowires are densely and uniformly distributed with typical length of nanowires around $10 \mu \mathrm{m}$. The successful fabrication of industrially-relevant sized $\mathrm{Co}_{3} \mathrm{O}_{4}$ nano-array monolithic catalysts using hydrothermal synthesis method here represents an important step toward the large scale production and potential application of nano-array based monolithic catalysts. 


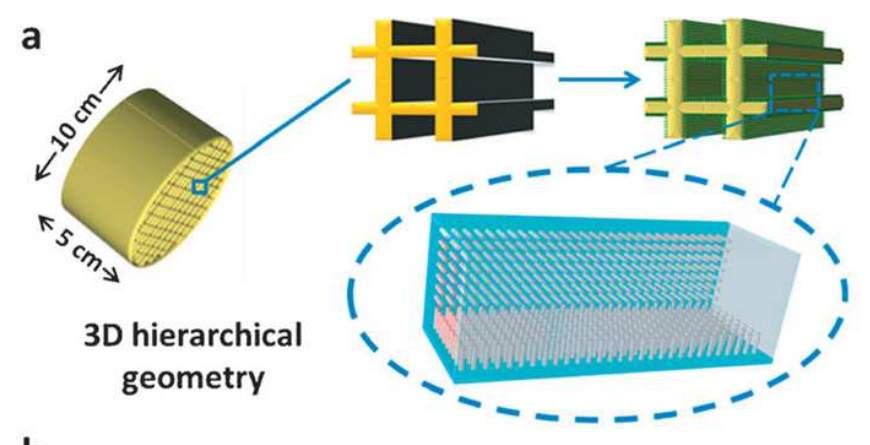

b
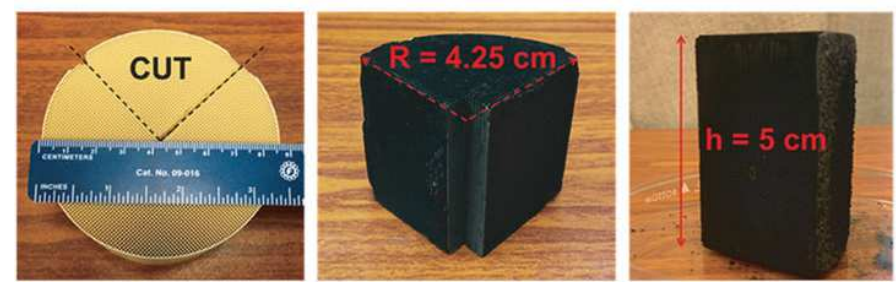

Figure 7. a) Schematic illustration of $\mathrm{Co}_{3} \mathrm{O}_{4}$ nano-array assembly on large cordierite monoliths; b) photographs of industrially-relevant sized monoliths before and after $\mathrm{Co}_{3} \mathrm{O}_{4}$ nano-array depostion. Reprinted with permission from ref. 31.
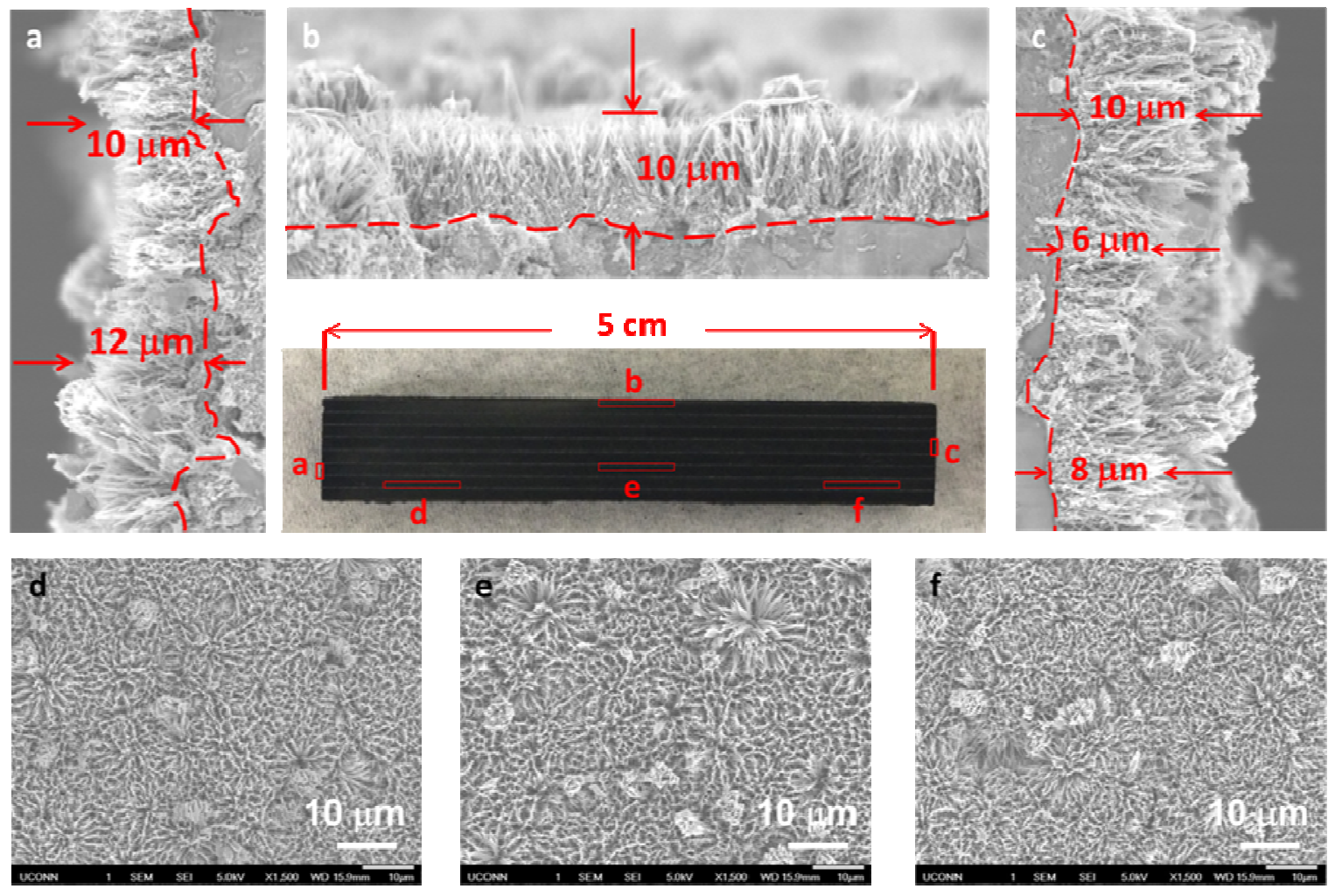
Figure 8. SEM characterization of $\mathrm{Co}_{3} \mathrm{O}_{4}$ nano-arrays grown upon different regions of the cordierite monolith channels. a-c) cross-sectional views and d-f) top views of $\mathrm{Co}_{3} \mathrm{O}_{4}$ nano-arrays. Reprinted with permission from ref. 31.

\section{Low Temperature Catalytic Oxidation Performance}

Commercial monolithic catalysts have been extensively used as catalytic converters for automotive emission control and industrial pollutant removal. With more and more stringent regulations placed upon the emission from vehicles and the waste stream from power plants, it is of great necessity to develop high performance catalyst for efficient automotive exhaust aftertreatment and clean energy combustion. Catalytic oxidation has so far been considered as a costeffective and efficient way to alleviate environmental pollution. For example, as the commercial three-way catalyst (TWC) equipped on gasoline engines, $\mathrm{Pt} / \mathrm{Rh}$ supported on $\gamma-\mathrm{Al}_{2} \mathrm{O}_{3}$ can simultaneously reduce $\mathrm{NO}_{\mathrm{x}}$ to nitrogen and convert $\mathrm{CO}$ and hydrocarbon $(\mathrm{HC})$ to $\mathrm{CO}_{2}$. In this section, we will discuss the performance of nano-array based monolithic catalysts toward several oxidation reactions at low temperature that are crucial for $\mathrm{CO}$ removal, $\mathrm{HCs}$ combustion and $\mathrm{NO}_{\mathrm{x}}$ abatement.

\subsection{CO oxidation}

Figure 9 demonstrates the catalytic $\mathrm{CO}$ oxidation performance of the hydrothermally grown monolithic nano-arrays loaded with Pt nanoparticles (1 wt.\% on nano-arrays). ${ }^{22}$ The size of these loaded Pt nanoparticles is $2 \mathrm{~nm}$ and they are well-dispersed on nanowires with the exposure of $\mathrm{Pt}$ $\{111\}$ atomic planes as identified by the inset HRTEM images. The catalyst activity toward CO oxidation was performed in the reaction feed of $1 \% \mathrm{CO}, 10 \% \mathrm{O}_{2}$ balanced by argon with a space velocity of $45,000 / \mathrm{h}$. The light-off temperatures $\mathrm{T}_{50}$ (defined as temperature at which $50 \%$ of CO conversion is achieved) for $\mathrm{CO}$ oxidation of Pt-loaded $\mathrm{CeO}_{2}, \mathrm{ZnO}, \mathrm{Co}_{3} \mathrm{O}_{4}$, and $\mathrm{TiO}_{2}$ nano-array monolithic catalysts are $193{ }^{\circ} \mathrm{C}, 260{ }^{\circ} \mathrm{C}, 195{ }^{\circ} \mathrm{C}$ and $258{ }^{\circ} \mathrm{C}$, respectively. All the nano-array based monolithic catalysts are able to achieve the complete $\mathrm{CO}$ below $300{ }^{\circ} \mathrm{C}$. The better $\mathrm{CO}$ oxidation performance exhibited in $\mathrm{Pt} / \mathrm{CeO}_{2}$ and $\mathrm{Pt} / \mathrm{Co}_{3} \mathrm{O}_{4}$ nano-array catalysts is due to the catalytic promotion effect of $\mathrm{Co}_{3} \mathrm{O}_{4}$ and $\mathrm{CeO}_{2}$ nanostructures with catalytic activity themselves. 

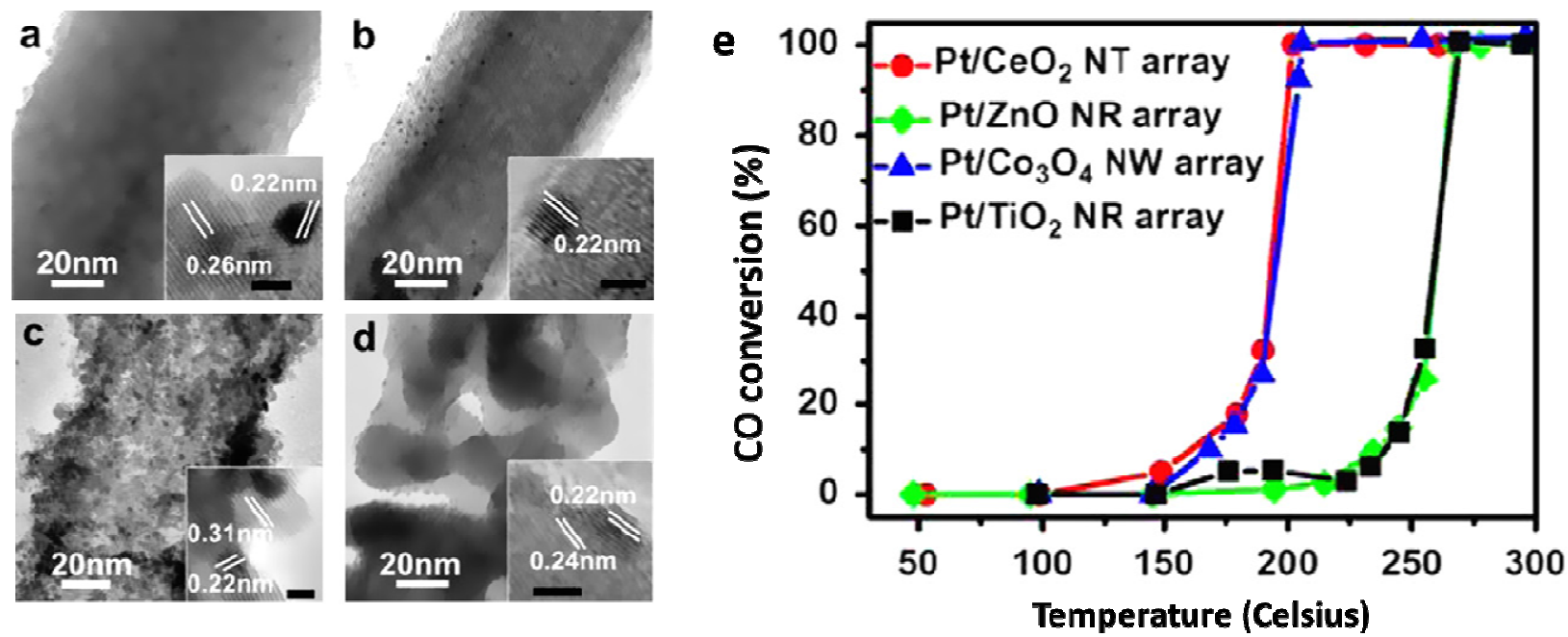

Figure 9. TEM images of $\mathrm{Pt}$ nanoparticles loaded on a) $\mathrm{ZnO}$ nanorod arrays; b) $\mathrm{TiO}_{2}$ nanorod arrays; c) $\mathrm{CeO}_{2}$ nanotube arrays and d) $\mathrm{Co}_{3} \mathrm{O}_{4}$ nanowire arrays; e) $\mathrm{CO}$ oxidation performances of $\mathrm{Pt} /$ metal oxide nano-arrays $\left(1 \% \mathrm{CO}, 10 \% \mathrm{O}_{2}\right.$, space velocity 45,000/h). Reprinted with permission from ref. 22 .

In addition to the Pt supported on metal oxides, the activity of metal oxide/perovskite nanoarrays has also been studied. We have successfully fabricated $\mathrm{TiO}_{2} /(\mathrm{La}, \mathrm{Sr}) \mathrm{MnO}_{3}$ composite nano-arrays on the planar substrates such as Si and glass by combined hydrothermal growth and magnetron sputtering. ${ }^{26}$ As shown in Figure 10a, aligned and densely packed $\mathrm{TiO}_{2} /(\mathrm{La}, \mathrm{Sr}) \mathrm{MnO}_{3}$ nanorods vertically grew on the substrate. The cross-sectional SEM image clearly shows the length of the composite nanorods $\sim 1 \mu \mathrm{m}$ and the diameter $\sim 30-100 \mathrm{~nm}$. The as-prepared composite nanorods were composed of single crystalline $\mathrm{TiO}_{2}$ nanorod core with amorphous perovskite shell, which can be turned into crystalline after thermal annealing at $800{ }^{\circ} \mathrm{C}$ for 3 hours. Figure 10c showed the typical single $\mathrm{TiO}_{2} /(\mathrm{La}, \mathrm{Sr}) \mathrm{MnO}_{3}$ composite nanorod after annealing, with a ridged ( $\mathrm{La}, \mathrm{Sr}) \mathrm{MnO}_{3}$ shell clearly observed. $\mathrm{TiO}_{2} /(\mathrm{La}, \mathrm{Sr}) \mathrm{MnO}_{3}$ core-shell nanorod array achieves $100 \% \mathrm{CO}$ conversion at $400{ }^{\circ} \mathrm{C}$, while pure $\mathrm{TiO}_{2}$ nanorod array just showed ca. 20\% CO conversion efficiency. The 100\% conversion temperature has been discovered to be lower than that of the reported powder form ( $\mathrm{La}, \mathrm{Sr}) \mathrm{MnO}_{3}$ catalysts. Both the high surface area enabled by the nano-array configuration and the oxygen vacancies of ( $\mathrm{La}, \mathrm{Sr}) \mathrm{MnO}_{3}$ film are responsible for the improved catalytic activity. Some other nano-array catalysts developed for $\mathrm{CO}$ oxidation includes $\mathrm{CuO}$ nano-arrays. ${ }^{32}$ 

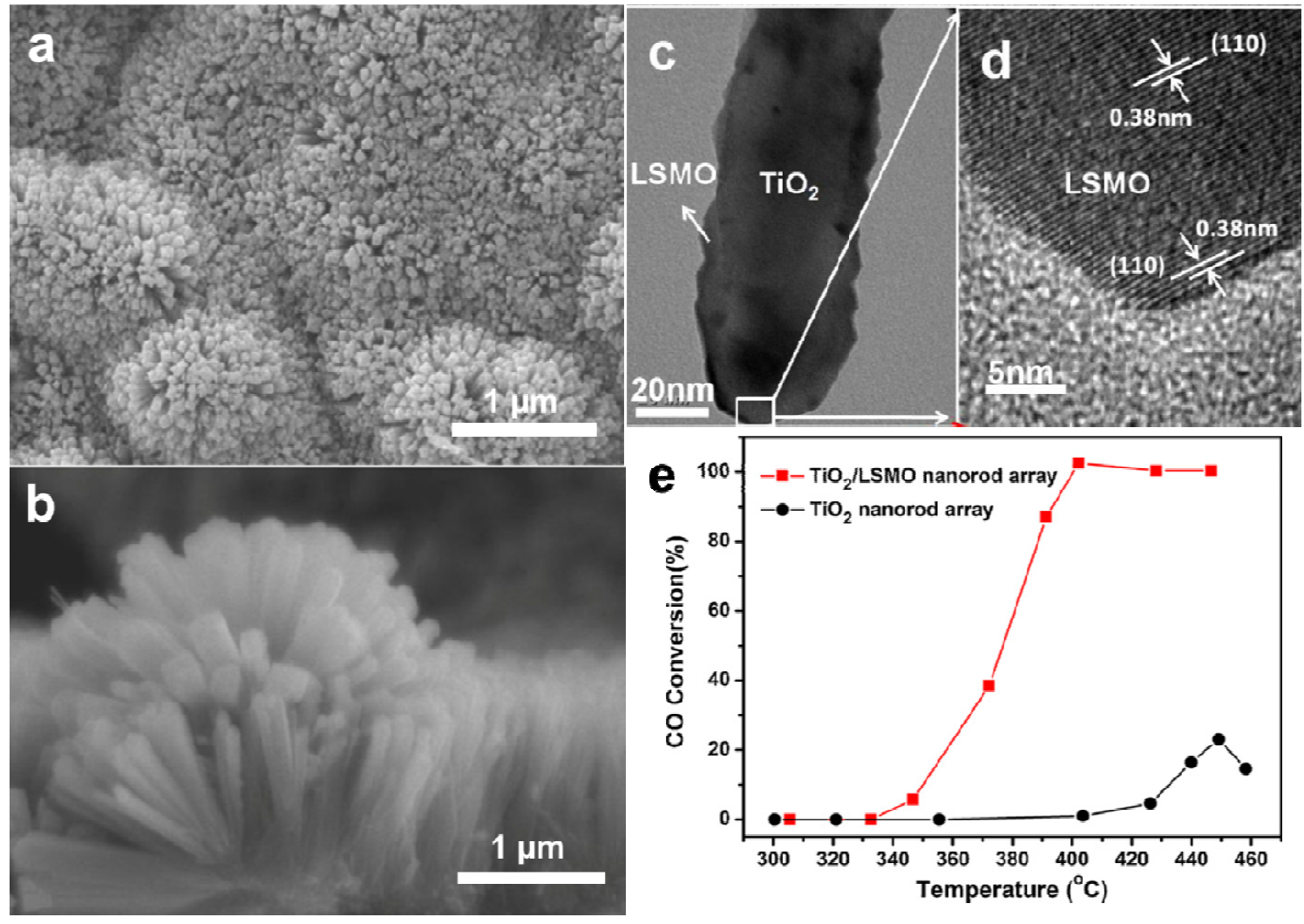

Figure 10. a) A top view SEM image of $\mathrm{TiO}_{2} /(\mathrm{La}, \mathrm{Sr}) \mathrm{MnO}_{3}$ nanorod arrays on Si substrates; b) a cross-sectional view of the $\mathrm{TiO}_{2} /(\mathrm{La}, \mathrm{Sr}) \mathrm{MnO}_{3}$ nanorod array; c) TEM image of a typical $\mathrm{TiO}_{2} /(\mathrm{La}, \mathrm{Sr}) \mathrm{MnO}_{3}$ core-shell nanorod after $800{ }^{\circ} \mathrm{C}$ annealing; d) TEM lattice image of ( $\mathrm{La}, \mathrm{Sr}) \mathrm{MnO}_{3}$ shell; e) Catalytic $\mathrm{CO}$ oxidation performance of $\mathrm{TiO}_{2} /(\mathrm{La}, \mathrm{Sr}) \mathrm{MnO}_{3}$ core-shell nanorod arrays and $\mathrm{TiO}_{2}$ nanorod arrays. Reaction conditions: $0.9 \times 0.45 \mathrm{~cm}^{2}$ substrate, $1 \%$ $\mathrm{CO} / 10 \% \mathrm{O}_{2} / \mathrm{Ar}$, flow rate: $30 \mathrm{ml} / \mathrm{min}$. Reprinted with permission from ref. 26 .

\subsection{NO oxidation}

Selective catalytic reduction (SCR) and $\mathrm{NO}_{\mathrm{x}}$ storage and reduction (NSR) are major chemical processes applicable for $\mathrm{NO}_{\mathrm{x}}$ abatement. For both technologies, the relatively high conversion of $\mathrm{NO}$ to $\mathrm{NO}_{2}$ is critical since $\mathrm{NO}_{2}$ is not only easier to be reduced by hydrocarbon or ammonia in the SCR process but favorable to be stored by barium oxide in NSR as well. In the past decade, much research effort has been focused on developing high performance and cost-effective catalysts for NO oxidation at low temperature. For example, strontium doped perovskites have been discovered to function as a high performance catalyst for $\mathrm{NO}_{\mathrm{x}}$ treatment with high catalytic activity toward low temperature $\mathrm{NO}$ to $\mathrm{NO}_{2}$ conversion. ${ }^{33}$ Recently, we have prepared 
monolithic $\mathrm{Co}_{3} \mathrm{O}_{4}$ nano-arrays which exhibit good $\mathrm{NO}$ oxidation performance at low temperature. ${ }^{34}$ The SEM images in Figure 11 show the uniform coverage of $\mathrm{Co}_{3} \mathrm{O}_{4}$ nano-arrays all around the cordierite monolith. The cobalt nitrate leads to $\mathrm{Co}_{3} \mathrm{O}_{4}$ nanowires of smaller diameter while cobalt chloride gives rise to thicker nanowires with sharp tips. The nano-arrays derived from cobalt chloride are distributed on the monolith channel surface in a more ordered fashion. The catalytic NO oxidation was performed by introducing the reaction feed of $500 \mathrm{ppm}$

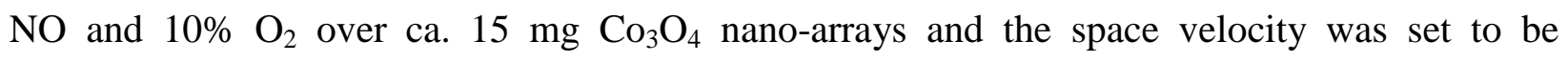
$\sim 50,000 / \mathrm{h}$. As shown in Figure 11c, the $\mathrm{Co}_{3} \mathrm{O}_{4}$ nano-arrays prepared from nitrate and acetate can achieve $80 \%$ NO to $\mathrm{NO}_{2}$ conversion at temperature below $300{ }^{\circ} \mathrm{C}$, which rivals thePt group metal catalysts. Moreover, the catalytic activity of nano-arrays was found to be better than that of the powder form catalyst prepared from the same hydrothermal process in terms of the NO conversion efficiency and the reaction temperature. The $\mathrm{Co}_{3} \mathrm{O}_{4}$ powder catalysts (same amount of loading) only achieve $60 \%$ conversion at temperature above $300{ }^{\circ} \mathrm{C}$, which further demonstrates the advantages of nano-array configuration over the conventional wash-coated catalysts.
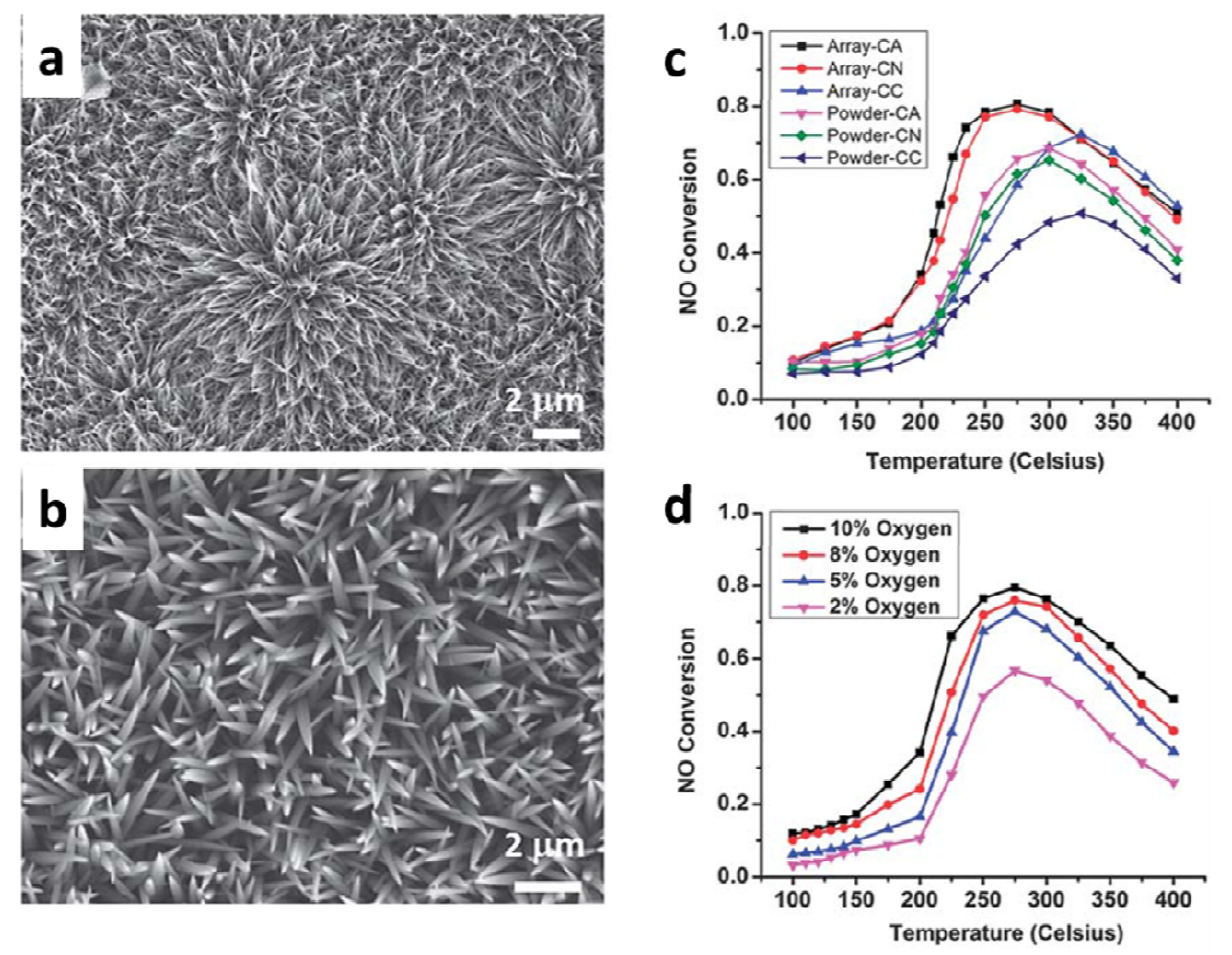

Figure 11. a-b) SEM images of monolithic $\mathrm{Co}_{3} \mathrm{O}_{4}$ nano-arrays prepared from cobalt nitrate and cobalt chloride, respectively; c) Comparison of catalytic NO oxidation performance of $\mathrm{Co}_{3} \mathrm{O}_{4}$ 
nano-arrays prepared from acetate, nitrate and chloride and the powder catalyst synthesized from the same hydrothermal process; d) $\mathrm{NO}$ oxidation performance over $\mathrm{Co}_{3} \mathrm{O}_{4}$ nano-array monolithic catalysts in $\mathrm{O}_{2}$ rich and deficient atmospheres. Reprinted with permission from ref. 34.

\subsection{Light alkane combustion}

Nano-array based monoliths can function as a promising reactor for the catalytic hydrocarbon combustion given their advantages including enhanced internal diffusion by ordered orientation, increased surface area of nanoscale dimension and thermal and mechanical stabilities. For example, $\mathrm{CuO}$ nanowire arrays were fabricated by directly annealing copper mesh at $520{ }^{\circ} \mathrm{C}$ for 2 days. $^{35}$ Figures $12 \mathrm{a}$ and $12 \mathrm{~b}$ display the photographs of the copper mesh before and after the annealing. It is clearly seen that the color changes from bright yellow to black indicating the oxidation to $\mathrm{CuO}$. The morphology and the orientation of the as-grown $\mathrm{CuO}$ nanowires were further examined by electron microscopy in Figures $12 \mathrm{c}$ and $12 \mathrm{~d}$. The $\mathrm{CuO}$ nanowires were formed perpendicularly to the copper mesh with an average diameter of $100 \mathrm{~nm}$ and length of 50 $\mu \mathrm{m}$ and they are uniformly distributed on the copper mesh. The catalytic activity of the $\mathrm{CuO}$ nanowires for $\mathrm{CH}_{4}$ oxidation was investigated with reaction feed of $1.5 \mathrm{vol}$. $\% \mathrm{CH}_{4}$ and 6 vol.\% $\mathrm{O}_{2}$ balanced by helium and the total flow rate was set to be $100 \mathrm{~mL} / \mathrm{min}$. It has been observed the catalytic activity is dependent on the nanowire length. With longer nanowires generated by prolonged reaction time, the $\mathrm{CuO}$ nano-arrays exhibit better performance with $\mathrm{CH}_{4}$ conversion as high as $40 \%$ at $500{ }^{\circ} \mathrm{C}$.

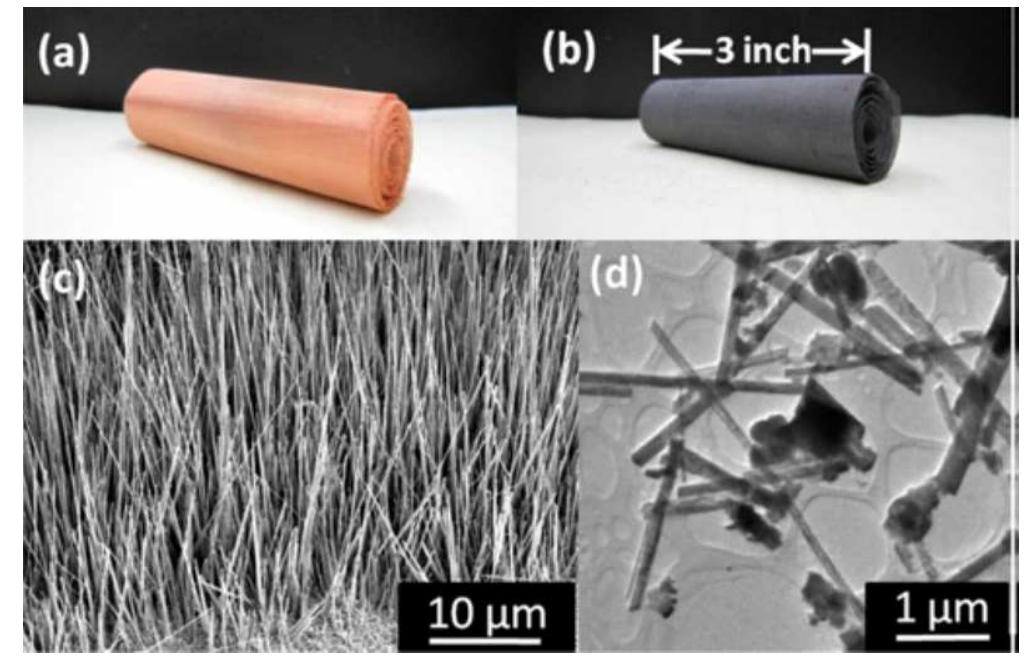


Figure 12. Photographs of (a) the copper mesh and (b) the annealed copper mesh $\left(520{ }^{\circ} \mathrm{C}, 2\right.$ days) grown with $\mathrm{CuO}$ nanowires. (c) SEM image and (d) TEM image of the as-grown $\mathrm{CuO}$ nanowires. Reprinted with permission from ref. 35.

We recently developed 2 inch long monolithic catalysts based on $\mathrm{Co}_{3} \mathrm{O}_{4}$ nano-arrays which shows excellent catalytic methane combustion performance with a low catalyst usage (ca. 15 $\mathrm{mg}){ }^{31}$ Figure 13 shows the catalytic methane oxidation performance of pristine and doped $\mathrm{Co}_{3} \mathrm{O}_{4}$ nano-arrays. The $\mathrm{Co}_{3} \mathrm{O}_{4}$ nano-arrays doped with $\mathrm{Ni}$ exhibit better activity at low temperature and can achieve complete combustion of $\mathrm{CH}_{4}$ below $600{ }^{\circ} \mathrm{C}$. Similar promotion effect by foreign atoms has been reported by other research groups. For example, Feng and co-workers directly grew $\mathrm{Co}_{3} \mathrm{O}_{4}$ nanowires arrays on stainless steel mesh substrate by ammonia-evaporation-induced growth. ${ }^{36}$ The addition of $\mathrm{Cu}^{2+}$ was found to promote the nanowires arrays growth as shown in Figure 14. The addition of $\mathrm{Cu}^{2+}$ was also discovered to enhance the catalyst activity for methane oxidation. These two examples demonstrate the monolithic nano-arrays catalyst based on transition metal oxide has great potential for low temperature hydrocarbon combustion and the associated activity may be promoted by introducing foreign ions in the synthesis. The rational design of catalyst with adjusted activity will be discussed later in the next section.
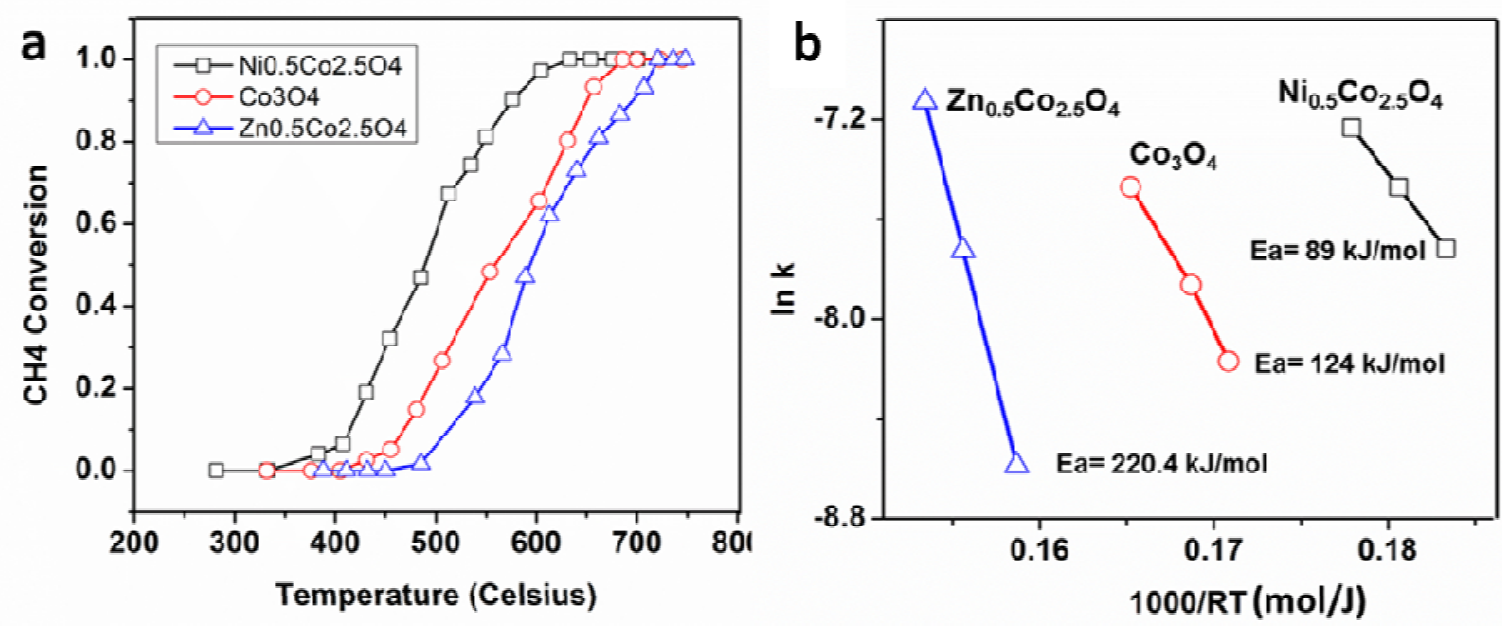

Figure 13. a) Catalytic $\mathrm{CH}_{4}$ oxidation performance of pristine and transition metal doped $\mathrm{Co}_{3} \mathrm{O}_{4}$ nano-arrays; b) Arrhenius plots for apparent activation energy calculation. Reprinted with permission from ref. 31 . 


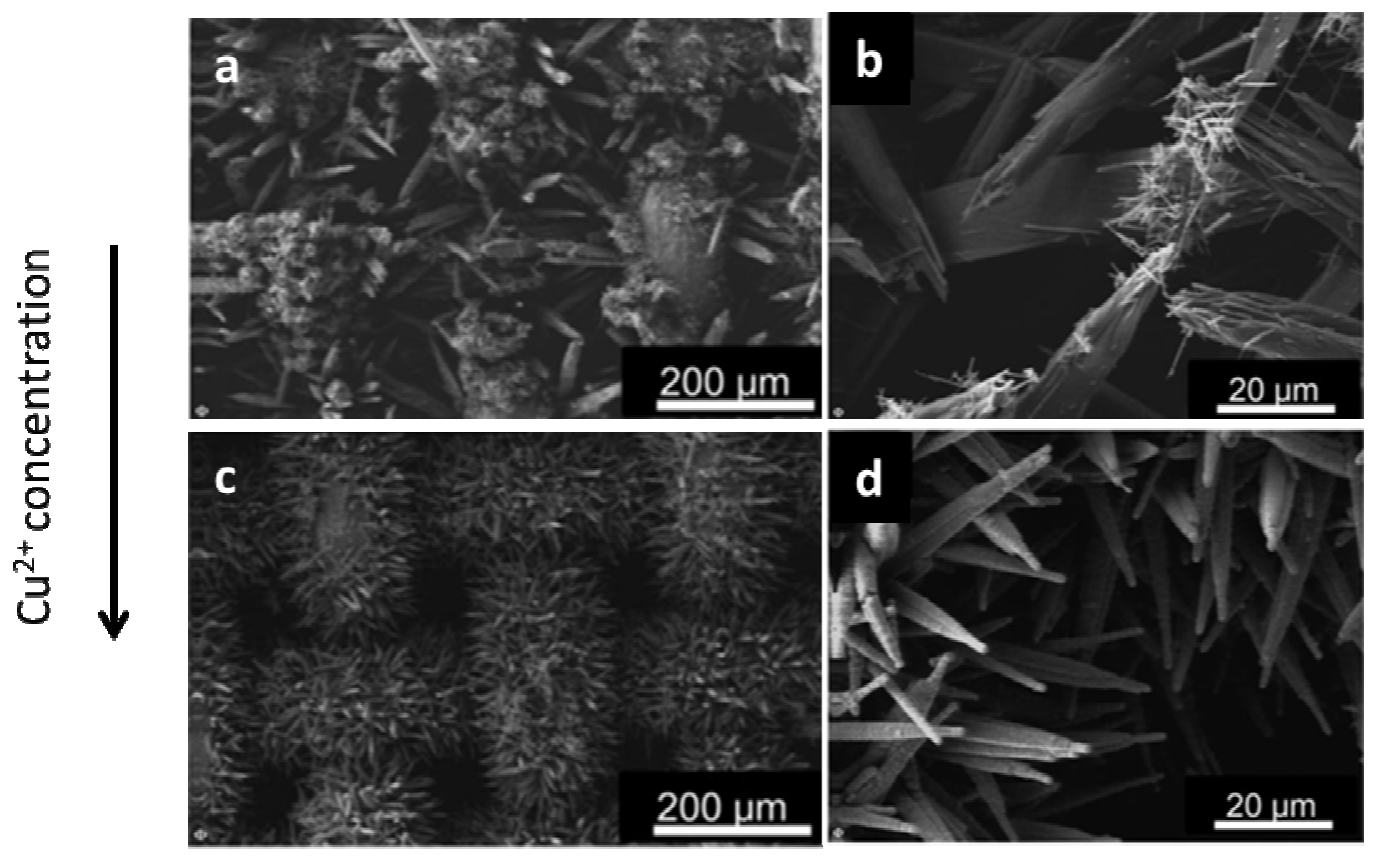

Figure 14. $\mathrm{Cu}$ ion effect on $\mathrm{Co}_{3} \mathrm{O}_{4}$ nano-array growth upon stainless steels indicating increased $\mathrm{Cu}$ concentration promotes nanowire growth. Reprinted with permission from ref.36.

\section{Tunable Catalytic Activity}

An important feature of monolithic nano-arrays is their well-defined geometry other than the random microstructure of the wash-coated particulates. This opens up the possibility to build precise and optimum structure-property correlations for catalysts in the materials science perspective. In this section we will introduce several examples that adjust catalyst performance by either tuning nano-array geometry or manipulating its chemical composition. We hope the discussion here could elucidate the structure-property relationship in nano-array catalysis and provide some insightful guidance of rational nano-array catalyst design for catalytic oxidation reactions.

\subsection{Size and shape dependence}

The nanowire array with well-defined geometry and orientation provides a good platform to study the structure-property relationship in nano-catalysis. With the rapid development of solution chemistry, it has become a relatively routine process to produce nanostructures with controlled shape, morphology and orientation. It has been widely reported the nanocrystals with different controlled geometry are able to exhibit different catalytic activity. ${ }^{37}$ For instance, $\mathrm{CeO}_{2}$ 
nanocrystals with different shapes have different dominant crystal planes exposed, which lead to different activity toward $\mathrm{CO}$ oxidation. ${ }^{38} \mathrm{CeO}_{2}$ nanorods of exposed $\{110\}$ and $\{100\}$ planes have better catalytic performance than that of nanocubes with mainly $\{100\}$ and octahedral nanocrystals with $\{111\}$ planes. Similar phenomenon was observed in the $\mathrm{Co}_{3} \mathrm{O}_{4}$ nanocrystals of different morphology. ${ }^{39,40} \mathrm{Co}_{3} \mathrm{O}_{4}$ nanosheets, nanobelts and nanocubes were successfully prepared by the hydrothermal process and the methane combustion catalytic activity order of crystal planes follows $\{112\}>\{011\} \gg\{001\} .{ }^{39}$ For the nano-array based monolithic catalysts, the size of the nanostructures can also be tuned in addition to the enclosed crystal planes. As an apt example, Pt loaded $\mathrm{ZnO}$ nano-arrays have been used to illustrate how the size and the crystal plane effects are able to adjust the catalytic performance. ${ }^{22}$ The catalytically inactive $\mathrm{ZnO}$ nanostructure as a support provides a good platform to exemplify the roles of its enclosed surfaces and interfaces with catalytically active Pt nanoparticles. We successfully tuned the aspect ratio of $\mathrm{ZnO}$ nano-arrays to achieve both nanowires with dominant side plane $\{10 \overline{1} 0\}$ and nanoplates with dominant polar surface $\{0001\}$. Furthermore, the length of $\mathrm{ZnO}$ nanowires can be increased from $1 \mu \mathrm{m}$ to $5 \mu \mathrm{m}$ by continuous growth with additional cycles. Figure 15 shows the electron microscopy images of $\mathrm{ZnO}$ nano-arrays with different morphology and different lengths in comparison with the wash-coated commercial $\mathrm{ZnO}$ powders. The same amount of $\mathrm{Pt}$ nanoparticles was loaded onto these different $\mathrm{ZnO}$ nano-arrays to investigate the structure effects of $\mathrm{ZnO}$ support on the catalytic performance. The light-off curves of different nano-array supported Pt nanoparticles are shown in Figure 16. It is clearly seen that Pt nanoparticles anchored on longer $\mathrm{ZnO}$ nanowires exhibit better activity and achieve complete $\mathrm{CO}$ conversion at lower temperature. However, the performance of $\mathrm{Pt} / \mathrm{ZnO}$ nanowires is not as good as that of $\mathrm{Pt}$ supported on $\mathrm{ZnO}$ nanoplates. The activity difference may be attributed to the interfacial promotion effects by the synergy between Pt nanoparticles and the polar $\mathrm{ZnO}\{0001\}$ surfaces which are predominant in $\mathrm{ZnO}$ nanoplates. It is worth noticing that the catalytic activity of $\mathrm{Pt}$ supported on all $\mathrm{ZnO}$ nano-arrays is better than that of $\mathrm{Pt} / \mathrm{ZnO}$ wash-coated powder. Therefore, by integrating $\mathrm{ZnO}$ nano-arrays with different geometry, the catalytic activity of monolithic nano-arrays can be rationally adjusted toward $\mathrm{CO}$ oxidation. By controlling and manipulating the nano-array catalyst and support structures, it opens up numerous possibilities to tune the collective catalyst performance. In the meantime, the above mentioned investigation can serve as 
a perfect example of selectively adjusting catalyst activity based on structure-property correlations.

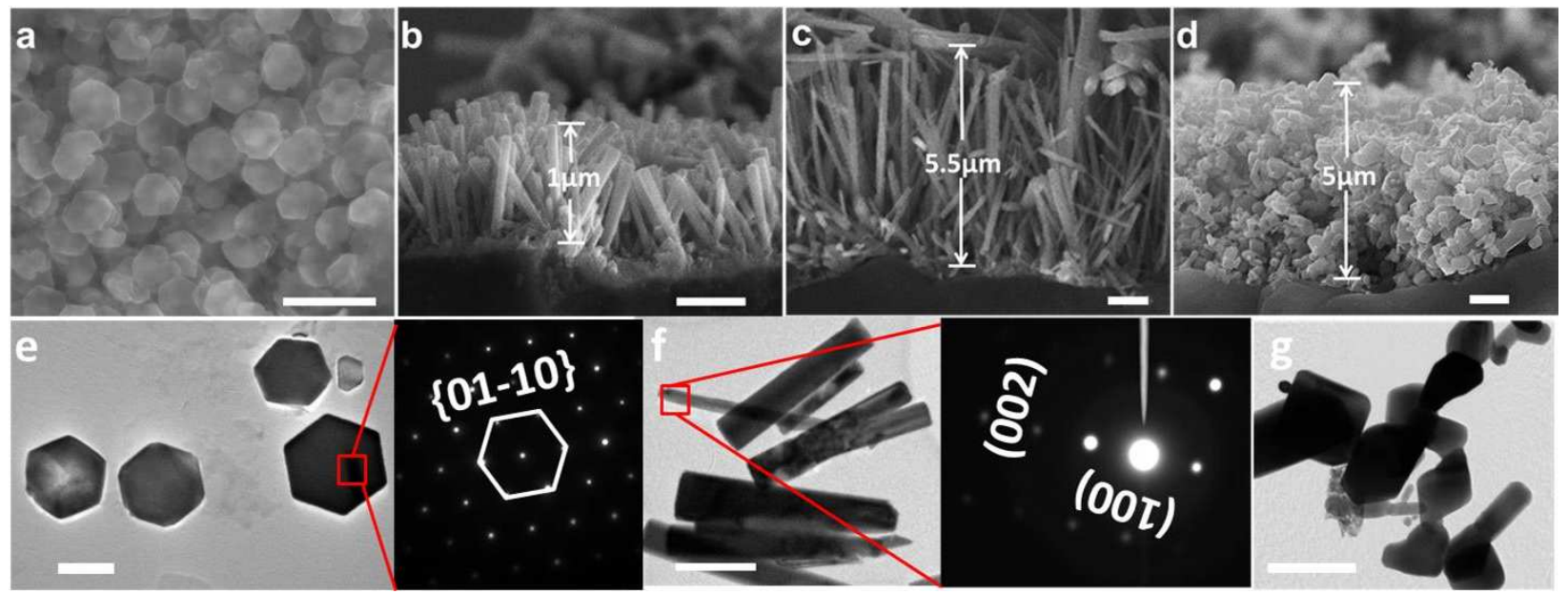

Figure 15. Electron microscopy characterization of $\mathrm{ZnO}$ nano-arrays with different shapes and sizes. SEM images of a) nanoplates; b) $1 \mu \mathrm{m}$ nanowire; c) $5 \mu \mathrm{m}$ nanowire; d) wash-coated $\mathrm{ZnO}$ powders. TEM images of e) $\mathrm{ZnO}$ nanoplate with inset electron diffraction pattern; f) $\mathrm{ZnO}$ nanowires with inset electron diffraction pattern; g) $\mathrm{ZnO}$ powders. Reprinted with permission from ref.22.
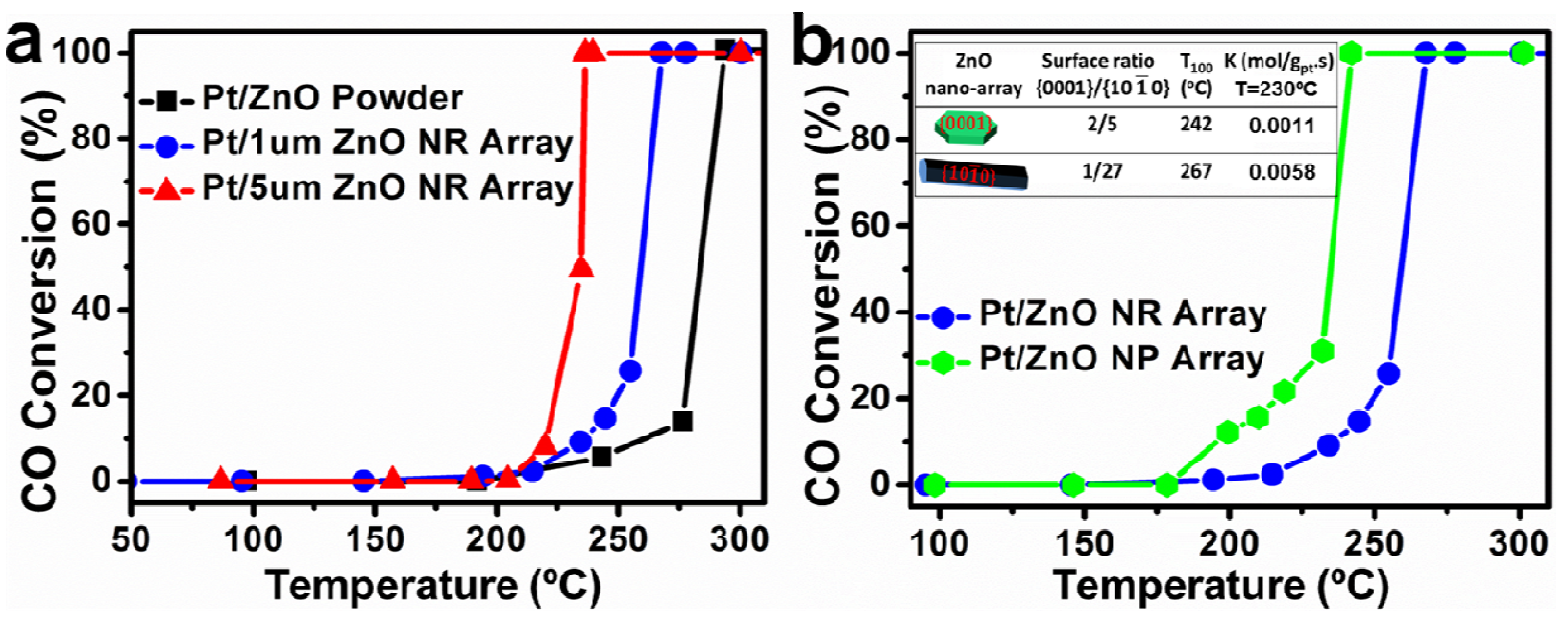

Figure 16. Catalytic $\mathrm{CO}$ oxidation performance of Pt loaded on $\mathrm{ZnO}$ nano-arrays with different lengths and shapes. Reprinted with permission from ref.22.

\subsection{Guest atoms and defects manipulation}


In addition to the geometry control and crystal plane manipulation, the catalytic activity of nanoarray based monolithic catalysts can also be modified by variation of chemical composition. ${ }^{31}$ As previously discussed, we have demonstrated in Figure 13 the incorporation of $\mathrm{Ni}$ into the spinel $\mathrm{Co}_{3} \mathrm{O}_{4}$ lattice leads to enhanced catalytic performance toward $\mathrm{CH}_{4}$ oxidation at low temperature, while the $\mathrm{Zn}$ doping seems not to promote reaction kinetics. TEM characterization was first employed to understand the nanocrystal structure. As shown in Figure 17, the porous $\mathrm{M}_{0.5} \mathrm{Co}_{2.5} \mathrm{O}_{4}(\mathrm{M}=\mathrm{Co}, \mathrm{Ni}$ and $\mathrm{Zn})$ nanowires all expose $\{110\}$ planes and therefore $\{110\}$ crystal plane was selected to perform the density functional theory (DFT) simulation to investigate the adsorption behavior of related species during the reactions. It has been proved by both DFT calculation and Raman spectroscopy analysis that $\mathrm{Ni}$ atoms take the octahedral Co sites in the spinel lattice while $\mathrm{Zn}$ atoms occupy the tetrahedral Co site. The corresponding doped $\{110\}$ surface model has been depicted in Figure 17e with top views of atom configurations. The DFT calculation results reveal the surface oxygen is responsible for $\mathrm{C}-\mathrm{H}$ activation in hydrocarbons by taking away $\mathrm{H}$. The $-\mathrm{CH}_{3}$ is stable on $\mathrm{Zn}$ while active on $\mathrm{Ni}$ site, which facilitates the further oxidation. So the Ni doping gives rise to the enhanced catalytic activity and thus lower combustion temperature. However, when $\mathrm{Co}$ is replaced by $\mathrm{Ni}$ and $\mathrm{Zn} \mathrm{CO}$ adsorption is no longer favored due to the less negative adsorption energy from DFT calculation, the CO oxidation performance is thus worsened by the doping. Based on the combined experimental observation and the DFT calculation that $\mathrm{Ni}$ promotes the $\mathrm{CH}_{4}$ oxidation, the concentration of $\mathrm{Ni}$ in the spinel $\mathrm{Co}_{3} \mathrm{O}_{4}$ lattice is further adjusted. As displayed in Figure 18, the increased Ni doping concentration is able to further promote reaction kinetics and push the complete $\mathrm{CH}_{4}$ conversion toward lower temperature. However, it is worth noting that the performed DFT calculation deals with clean surface without the defects. The $\mathrm{Ni}$ incorporation also generates surface oxygen defects since $\mathrm{Ni}^{2+}$ occupies octahedral $\mathrm{Co}\left(\mathrm{Co}^{3+}\right)$ sites leading to the positive charges loss. ${ }^{41}$ Such charge loss is compensated by oxygen vacancy formation to maintain the charge neutrality. XPS spectra in Figure 18a therefore reveal the deconvoluted $\mathrm{O}$ 1s signals and indicate defective oxygen population increases as the $\mathrm{Ni}$ concentration is increased. The rich oxygen defects on the surface are believed to enhance the surface lattice oxygen mobility since the bonding is no longer saturated. The enhanced oxygen mobility is able to promote $\mathrm{C}-\mathrm{H}$ activation and thus improve the reaction kinetics. 

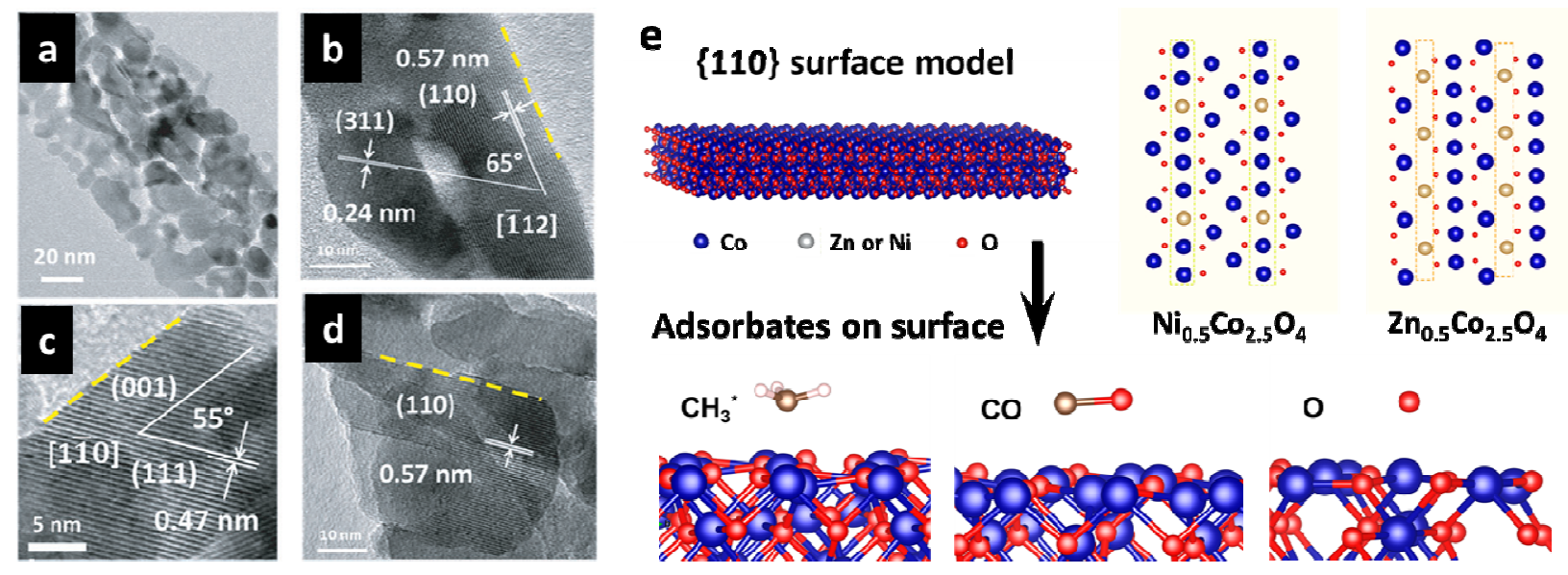

Figure 17. TEM images of a) and c) $\mathrm{Co}_{3} \mathrm{O}_{4}$ nanowires; b) $\mathrm{Ni}_{0.5} \mathrm{Co}_{2.5} \mathrm{O}_{4}$ nanowires; d) $\mathrm{Zn}_{0.5} \mathrm{Co}_{2.5} \mathrm{O}_{4}$ nanowires. Density functional theory simulation of the adsorption behaviors of surface species on $\mathrm{M}_{0.5} \mathrm{Co}_{2.5} \mathrm{O}_{4}\left(\mathrm{M}=\mathrm{Co}, \mathrm{Ni}\right.$ and $\mathrm{Zn}$ ) \{110\} surfaces for $\mathrm{CH}_{4}$ and $\mathrm{CO}$ oxidation. Reprinted with permission from ref. 31.
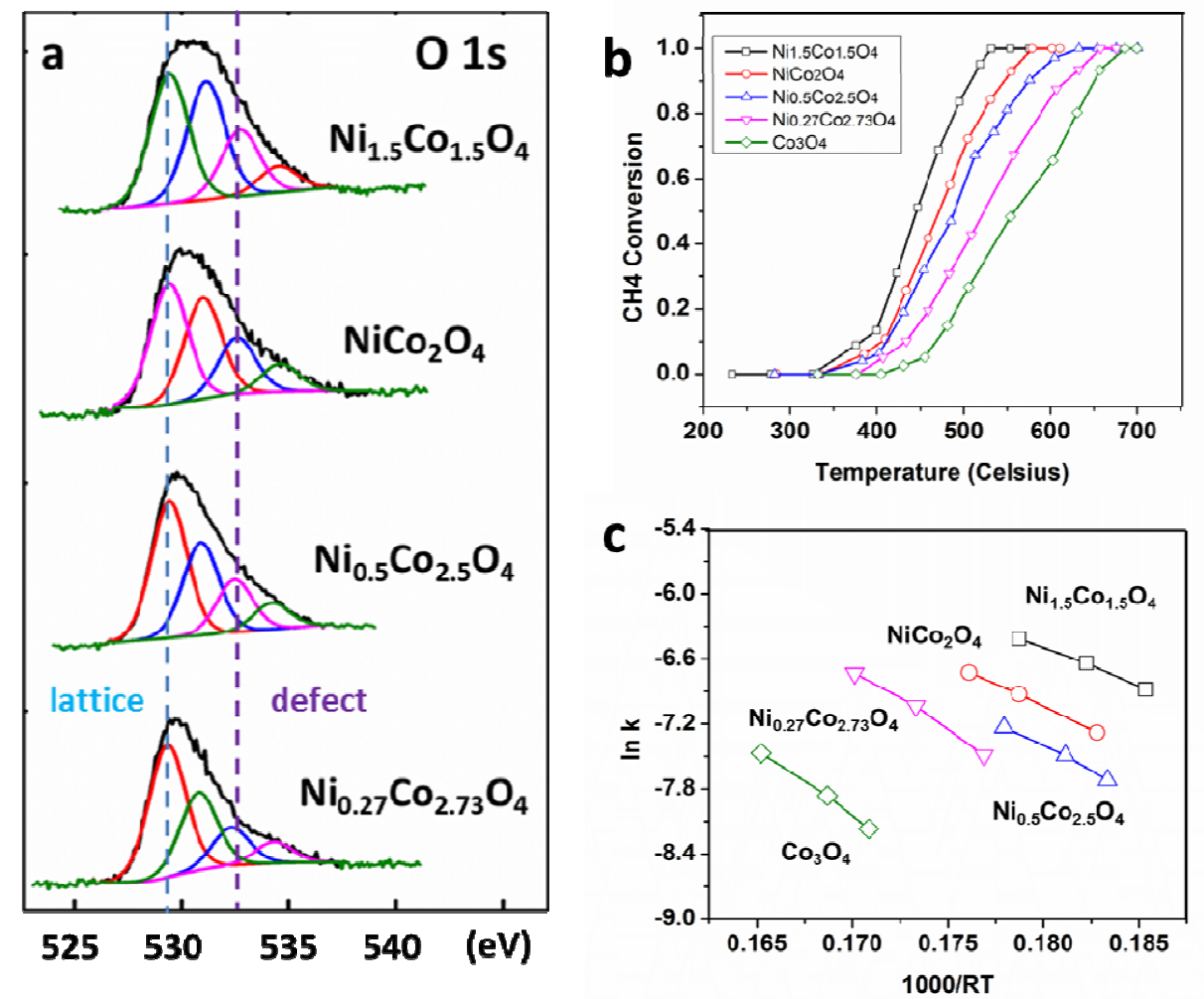

Figure 18. a) Deconvoluted XPS spectra of $\mathrm{O} 1 \mathrm{~s}$ for $\mathrm{Ni}$ doped $\mathrm{Co}_{3} \mathrm{O}_{4}$ with different $\mathrm{Ni}$ concentrations; b) catalytic $\mathrm{CH}_{4}$ oxidation performance of $\mathrm{Ni}$ doped $\mathrm{Co}_{3} \mathrm{O}_{4}$ nano-arrays; c) the corresponding Arrhenius plots revealing the reaction kinetics. Reprinted with permission from ref. 31 . 


\section{Summary and Perspectives}

In this review, we start with the discussion of monolithic catalysts and illustrate the advantages of monolithic configuration, the existing challenges associated with the catalyst preparation and the difficulty of relating industrial catalyst structure to its catalytic property. To mitigate and address these issues, we have proposed and demonstrated a new monolithic catalyst design by replacing thick wash-coated catalyst layers with much thinner but ordered nano-arrays. These nano-arrays are in-situ grown uniformly throughout the monolithic substrate channel surfaces via a facile hydrothermal synthesis strategy. Several features and advantages of nano-array based monolithic catalyst over powder wash-coated monolithic catalyst are summarized as follows.

- Good uniformity and controlled nano-array thickness as well as free of binders

- High materials utilization efficiency with an order of magnitude reduction of catalyst usage (both oxide support and noble metals) without catalytic performance sacrificed

- Good thermal and mechanical stabilities, small surface area and weight loss during prolonged durations of high temperature aging and high velocity gas flux

- Well-defined catalyst structures with controlled geometry and orientation, providing a good platform to understand the exact structure-property relationship of monolithic catalyst

- Adjustable catalytic activity by manipulating nanostructure size and shape as well as controlled chemical composition.

In the later context, we demonstrate the 3D integration of nano-arrays and further introduce the scalable fabrication of nano-array based monolithic catalyst to the industry-relevant scale by addressing the mass transfer issue via the introduced mechanical agitation. Excellent catalytic performances of monolithic nano-arrays catalyst have been demonstrated using different nanoarray catalysts for various important oxidation reactions in automotive emission and clean energy combustion, such as $\mathrm{CO}$ oxidation, $\mathrm{NO}$ oxidation and hydrocarbon combustion. It has also been proved the careful adjustment of nano-array size and morphology is able to achieve tunable catalytic activity, which is crucial for rational design of efficient monolithic catalyst.

It is worth pointing out that the selected metal oxide nano-arrays demonstrated here are not intended as the best options as either supports or catalytically active materials for the low 
temperature catalytic reactions. Instead, the selected metal oxide systems are used as the model systems for us to better understand and design the new class of nano-array based structured catalysts with better performance and functions for various energy and environmental applications. Moreover, there are several major challenges for the nano-array based monolithic catalysts. First, the current solution chemistry strategy only applies in metal oxide catalysts which usually suffer from low hydrothermal stability and sulfur poisoning. The synthetic approaches for nano-arrays of several other catalytic materials such as zeolite and perovskite, which are more stable and widely used in catalysis industry, needs to be developed. Second, although the nano-array based monolithic catalysts demonstrate good catalytic activity toward some oxidation reactions, it is necessary to further improve the activity without increasing the catalyst usage. It thus means better understanding of the gas-solid interaction which determines the reaction kinetics in the nano-array configuration needs to be extracted. Third, the catalyst is required to perform multiple catalytic conversions since multiple gas components usually exist in industrial practice. For each specific industrial application the catalytic materials selection regarding the nano-array configuration, activity and stability is a nontrivial task. Our future research would be focused to mitigate these difficulties and provide possible solutions to address these challenges.

1. Materials selection and synthesis: design and develop effective strategies to fabricate and manufacture the nano-array morphology of more material systems including other oxides, perovskites and noble metals.

2. Catalytic reactions: apply nano-array based monolithic catalysts for complex reactions or chemical processes in industry and generate database of reaction kinetics, catalyst efficiency and potential hurdles.

3. Multifunctional catalyst design: rationally design nano-array catalysts with well-defined physical and chemical characteristics for concurrent multiple reactions.

4. Stability and sulfur tolerance: test and improve the hydrothermal, thermal and catalytic stabilities as well as the sulfur tolerance of monolithic nano-array catalyst.

We hope this article not only provides a comprehensive overview of the fabrication, advantages and performance of the newly invented nano-array based monolithic catalysts, but also serves as a timely and useful guidance to rational nanocatalysts design. 
Acknowledgement: The authors are grateful for the financial support from the US Department of Energy (Award \# DE-EE0006854) and the US National Science Foundation (Award \# CBET-

1344792). Z. R. would like to acknowledge the partial support from a General Electrics Graduate Fellowship for Innovation.

\section{Reference}

(1) Cybulski, A.; Moulijn, J. A. Catalysis Reviews 1994, 36, 179.

(2) Irandoust, S.; Andersson, B. Catalysis Reviews 1988, 30, 341.

(3) Williams, J. L. Catalysis Today 2001, 69, 3.

(4) Boger, T.; Heibel, A. K.; Sorensen, C. M. Industrial \& Engineering Chemistry Research $2004,43,4602$.

(5) Tomašić, V.; Jović, F. Applied Catalysis A: General 2006, 311, 112.

(6) Geus, J. W.; van Giezen, J. C. Catalysis Today 1999, 47, 169.

(7) Zamaro, J. M.; Ulla, M. A.; Miró, E. E. Chemical Engineering Journal 2005, 106, 25.

(8) Nijhuis, T. A.; Kreutzer, M. T.; Romijn, A. C. J.; Kapteijn, F.; Moulijn, J. A. Chemical Engineering Science 2001, 56, 823.

(9) Casanovas, A.; de Leitenburg, C.; Trovarelli, A.; Llorca, J. Catalysis Today 2008, 138, 187.

(10) Twigg, M. V. Applied Catalysis B: Environmental 2007, 70, 2.

(11) Heck, R. M.; Farrauto, R. J. Applied Catalysis A: General 2001, 221, 443.

(12) Heck, R. M.; Gulati, S.; Farrauto, R. J. Chemical Engineering Journal 2001, 82, 149.

(13) Adler, J. International Journal of Applied Ceramic Technology 2005, 2, 429.

(14) Yang, J.; Stewart, M.; Maupin, G.; Herling, D.; Zelenyuk, A. Chemical Engineering Science 2009, 64, 1625.

(15) Park, J.-K.; Park, J.-H.; Park, J.-W.; Kim, H.-S.; Jeong, Y.-I. Separation and Purification Technology 2007, 55, 321.

(16) Yao, Y.; Ochiai, T.; Ishiguro, H.; Nakano, R.; Kubota, Y. Applied Catalysis B: Environmental 2011, 106, 592.

(17) Park, J.; Lee, J.; Lee, S. Journal of Porous Materials 2002, 9, 203.

(18) Dong, Y.; Liu, X.; Ma, Q.; Meng, G. Journal of Membrane Science 2006, 285, 173.

(19) Zhou, L.; Wang, T.; Nguyen, Q. T.; Li, J.; Long, Y.; Ping, Z. Separation and Purification Technology 2005, 44, 266.

(20) Shigapov, A. N.; Graham, G. W.; McCabe, R. W.; Paputa Peck, M.; Kiel Plummer Jr, H. Applied Catalysis A: General 1999, 182, 137.

(21) Nijhuis, T. A.; Beers, A. E. W.; Vergunst, T.; Hoek, I.; Kapteijn, F.; Moulijn, J. A. Catalysis Reviews 2001, 43, 345.

(22) Guo, Y.; Ren, Z.; Xiao, W.; Liu, C.; Sharma, H.; Gao, H.; Mhadeshwar, A.; Gao, P.-X. Nano Energy 2013, 2, 873.

(23) Sun, J.; Li, Y.; Liu, X.; Yang, Q.; Liu, J.; Sun, X.; Evans, D. G.; Duan, X. Chemical Communications 2012, 48, 3379.

(24) Ren, Z.; Guo, Y.; Liu, C.-H.; Gao, P.-X. Frontiers in Chemistry 2013, 1. 
(25) Jian, D.; Gao, P.-X.; Cai, W.; Allimi, B. S.; Pamir Alpay, S.; Ding, Y.; Wang, Z. L.; Brooks, C. Journal of Materials Chemistry 2009, 19, 970.

(26) Guo, Y.; Zhang, Z.; Ren, Z.; Gao, H.; Gao, P.-X. Catalysis Today 2012, 184, 178.

(27) Xu, S.; Wang, Z. Nano Research 2011, 4, 1013.

(28) Guo, Y.; Liu, G.; Ren, Z.; Piyadasa, A.; Gao, P.-X. CrystEngComm 2013, 15, 8345.

(29) Zhang, Z.; Gao, H.; Cai, W.; Liu, C.; Guo, Y.; Gao, P.-X. Journal of Materials Chemistry 2012, 22, 23098.

(30) Xiao, W.; Guo, Y.; Ren, Z.; Wrobel, G.; Ren, Z.; Lu, T.; Gao, P.-X. Crystal Growth \& Design 2013, 13, 3657.

(31) Ren, Z.; Botu, V.; Wang, S.; Meng, Y.; Song, W.; Guo, Y.; Ramprasad, R.; Suib, S. L.; Gao, P.-X. Angewandte Chemie International Edition 2014, 53, 7223.

(32) Feng, Y.; Zheng, X. Nano Letters 2010, 10, 4762.

(33) Kim, C. H.; Qi, G.; Dahlberg, K.; Li, W. Science 2010, 327, 1624.

(34) Ren, Z.; Guo, Y.; Zhang, Z.; Liu, C.; Gao, P.-X. Journal of Materials Chemistry A 2013, 1, 9897.

(35) Feng, Y.; Rao, P. M.; Kim, D. R.; Zheng, X. Proceedings of the Combustion Institute 2011, $33,3169$.

(36) Feng, Y.; Zheng, X. ChemCatChem 2012, 4, 1551.

(37) Xie, X. W.; Li, Y.; Liu, Z. Q.; Haruta, M.; Shen, W. J. Nature 2009, 458, 746.

(38) Wu, Z.; Li, M.; Overbury, S. H. Journal of Catalysis 2012, 285, 61.

(39) Hu, L.; Peng, Q.; Li, Y. Journal of the American Chemical Society 2008, 130, 16136.

(40) Hu, L.; Sun, K.; Peng, Q.; Xu, B.; Li, Y. Nano Research 2010, 3, 363.

(41) Iliev, M. N.; Silwal, P.; Loukya, B.; Datta, R.; Kim, D. H.; Todorov, N. D.; Pachauri, N.; Gupta, A. Journal of Applied Physics 2013, 114. 